\title{
Role of Ultrasound in Thyroid Pathologies
}

\author{
Dr. Awad Mohamed Elkhadir \\ Department of Diagnostic Radiology-Faculty of Applied Medical Sciences King Abdulaziz University Saudi \\ Arabia
}

\begin{abstract}
This is a retrospective study of thyroid patients referred for ultrasound for the period of 2007to 2009 at different hospitals and dispensaries in the Kingdom of Saudi Arabia (Eastern area).

Importance of the study is; to create a reliable reference for the role of ultrasound in thyroid pathologies in King Saudi Arabia.

Study aimed to detect the Ultrasonic Differential Diagnosis of Thyroid pathologies.

This study was carried out on 303 patients of various types of thyroid diseases in King Saudi Arabia.232 (77\%) were female patients and 71(23\%) were males.

Along this series there were $25 \%$ of enlarged thyroid gland which act as the most common ultrasound findings of thyroid disorders followed by, $23 \%$ solitary thyroid nodules, $10 \%$ multinodular goiters, $8 \%$ simple cyst, 7

$\%$ nonpalpable thyroid nodules, $4 \%$ goiter and Hashimoto's thyroiditis.

The most common ultrasound findings of thyroid gland enlarged of thyroid.

The least common is nodal enlargement, adenoma, thyroiditis, microcalcifications, small size thyroid, multiple thyroid nodules, thyroglossal cyst and Graves disease.

Out of this study we believe more that ultrasound should be the first-line test owing to its safety and availability.

Keywords- Sonographic, Thyroid Gland, Thyroid Pathology, Ultrasound.
\end{abstract}

\section{Introduction}

Thyroid US is the imaging method of choice for the evaluation of thyroid gland structure, and FNAC, as the most accurate test for nodule diagnosis, has reduced the need for scanning and for thyroidectomy, thereby reducing the health-care costs significantly.

US results alone cannot be accepted as true positives in terms of malignancy. However, the procedure's usefulness is considerable, if combined with the clinical data and laboratory test results. As thyroid nodules are rare disorders the use of US examination is not contraindicated on economic grounds. Moreover, it helps to complete the diagnostic protocol of a thyroid nodule and subsequently to choose the best mode of treatment. US is a safe and widely available technique, and I therefore recommend it strongly as the first-line screening diagnostic test in all pediatric patients with thyroid nodules. This should be followed by further imaging-directed tests (SC (invariably in patients with suppressed TSH) and FNAC or direct FNAC in cystic (unechogenic) lesions).

Preoperative US examination of thyroid nodules not only provides information on their size, echogenecity, echo structure and location but also contributes significantly to the differential diagnosis of benign vs. malignant tumors. It is a simple, inexpensive and radiation-free method of examination of great sensitivity and specificity and is complementary to FNAC.Color-Doppler sonography may be helpful in hyper functioning nodules (hot on $\mathrm{SC}$ and usually benign on histology), indicating an intensive vascular flow within a highly vascularized lesion, and no visible flow through the remaining, suppressed thyroid gland. Color-Doppler sonography is also valuable in distinguishing a cystic lesion (with no vascular flow) from a solid neoplasm (with intranodular flow).Cystic degeneration occurring in previously solid lesions does not determine the diagnosis, whether benign or malignant. If there is no vascular network within the nodule, the nodule is painful on palpation and the patient has a fever, then a suppurative thyroiditis is suspected. US-guided FNAC, with a subsequent cytological examination and culture of the aspirated material, helps to identify a bacterial cause of the nodule.

US plays an important role in the diagnostic work-up of thyroid nodules. However, there are still doubts as to whether it is a sufficiently accurate method in the differentiation between benign and malignant lesions and therefore some deny its usefulness. On the other hand, authors from regions affected by radioactive fallout are convinced that systematic US screening is a significant tool for the early detection of thyroid carcinoma due to the many indicators of the malignant process which may be detected.

\subsection{Area of study: \\ Different Hospital and Dispensary for e.g.: \\ $\rightarrow$ Dammam Central Hospital. \\ $\rightarrow$ Fahad King Specialist Hospital.}




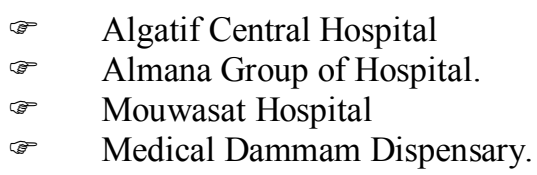

\subsection{Patients and methods:}

In order to be lying out, this research shall follow the scientific methods to collect the related information from different sources such as references, textbooks, Internet service, scientific magazines and data collection sheet. The study will be done in the hospitals mentioned above; the study will evaluate different patients, using real time machines with $7 \mathrm{MHz}$ linear array.

\subsection{Population sample}

The target populations for this research all thyroid ultrasound patients.

\subsection{Instrumentation}

Different types to ultrasound machines with $7 \mathrm{MHz}$ probe.

\subsection{Facilities available at hospital:}

Almana General Hospitals consist of four private hospitals in the Eastern province of Saudi Arabia, their capacity are 500 beds and equipped with 2 CT scans and one shimadzu, 6 Ultrasound Machines, two Ultramark 4, one Ultra mark 5 and one Ultra mark 9, one Apogee 800 and one Medison.

\section{The facilities available in this study also include:}

\section{2- Voluson 730 (GE Kretz) V 730 3D $\backslash 4 \mathrm{D}$}

Philips ATL HDI. 3000

GE. Logic 9

Philips EN Visor.

ALOKA SSD - 500

\section{Methodology:}

The way followed to collect the data in this study was data collecting sheet

\section{TABLES AND GRAPHS}

\section{Results}

Table (4-1): The sex distribution in between 303 cases with different sexes through this study illustrated in the table below:

\begin{tabular}{|l|l|l|}
\hline Patient's sex & Number of patients & Percentages \\
\hline Male & 71 & $23 \%$ \\
\hline Female & 232 & $77 \%$ \\
\hline Total & 303 & $100 \%$ \\
\hline
\end{tabular}

Summarize

\section{Case Processing Summary(a)}

\begin{tabular}{|r|r|r|r|r|r|r|}
\hline & \multicolumn{9}{|c|}{ Cases } \\
\cline { 2 - 7 } & & Included & \multicolumn{2}{|c|}{ Excluded } & Total \\
\cline { 2 - 7 } & $\mathrm{N}$ & Percent & $\mathrm{N}$ & Percent & $\mathrm{N}$ & Percent \\
\hline NoOfPatients & 3 & $100.0 \%$ & 0 & $.0 \%$ & 3 & $100.0 \%$ \\
Percentage & 3 & $100.0 \%$ & 0 & $.0 \%$ & 3 & $100.0 \%$ \\
\hline
\end{tabular}

a Limited to first 100 cases. 


\section{Case Summaries(a)}

\begin{tabular}{|c|c|c|c|}
\hline & & NoOfPatients & Percentage \\
\hline \multirow{8}{*}{ Total } & 1 & 71.00 & .23 \\
\hline & 2 & 232.00 & .77 \\
\hline & 3 & 303.00 & 1.00 \\
\hline & $\mathbf{N}$ & 3 & 3 \\
\hline & Mean & 202.0000 & .6667 \\
\hline & Std. Deviation & 118.87388 & .39526 \\
\hline & Range & 232.00 & .77 \\
\hline & Std. Error of Skewness & 1.225 & 1.225 \\
\hline
\end{tabular}

a Limited to first 100 cases.

Descriptive

Descriptive Statistics

\begin{tabular}{|r|r|r|r|r|r|}
\hline & $\mathrm{N}$ & Minimum & Maximum & Mean & Std. Deviation \\
\hline NoOfPatients & 3 & 71.00 & 303.00 & 202.0000 & 118.87388 \\
Percentage & 3 & .23 & 1.00 & .6667 & .39526 \\
Valid N (listwise) & 3 & & & & \\
\hline
\end{tabular}

T-Test

One-Sample Statistics

\begin{tabular}{|r|r|r|r|r|}
\hline & & & & Std. Error \\
& $\mathrm{N}$ & Mean & Std. Deviation & Mean \\
\hline NoOfPatients & 3 & 202.0000 & $\mathbf{1 1 8 . 8 7 3 8 8}$ & $\mathbf{6 8 . 6 3 1 8 7}$ \\
Percentage & 3 & .6667 & .39526 & .22821 \\
\hline
\end{tabular}

One-Sample Test

\begin{tabular}{|c|c|c|c|c|c|c|}
\hline & \multicolumn{6}{|c|}{ Test Value $=0$} \\
\hline & \multirow[b]{2}{*}{$\mathbf{t}$} & \multirow[b]{2}{*}{ df } & \multirow[b]{2}{*}{ Sig. (2-tailed) } & \multirow[b]{2}{*}{$\begin{array}{r}\text { Mean } \\
\text { Difference } \\
\end{array}$} & \multicolumn{2}{|c|}{$\begin{array}{r}95 \% \text { Confidence Interval of } \\
\text { the Difference }\end{array}$} \\
\hline & & & & & Lower & Upper \\
\hline NoOfPatients & 2.943 & 2 & .099 & 202.00000 & -93.2991 & 497.2991 \\
\hline Percentage & 2.921 & 2 & .100 & .66667 & -.3152 & 1.6486 \\
\hline
\end{tabular}

Two-stage Least Squares Analysis Model Description

\begin{tabular}{|rr|r|}
\hline & Type of Variable \\
\hline Equation 1 & Percentage & Dependent \\
& NoOfPatients & predictor \& instrumental \\
\hline
\end{tabular}

Model Summary

MOD_8

\begin{tabular}{|r|r|}
\hline Equation 1 & Multiple R \\
R Square & 1.000 \\
Adjusted R Square & 1.000 \\
Std. Error of the Estimate & 1.000 \\
& .004 \\
\hline
\end{tabular}




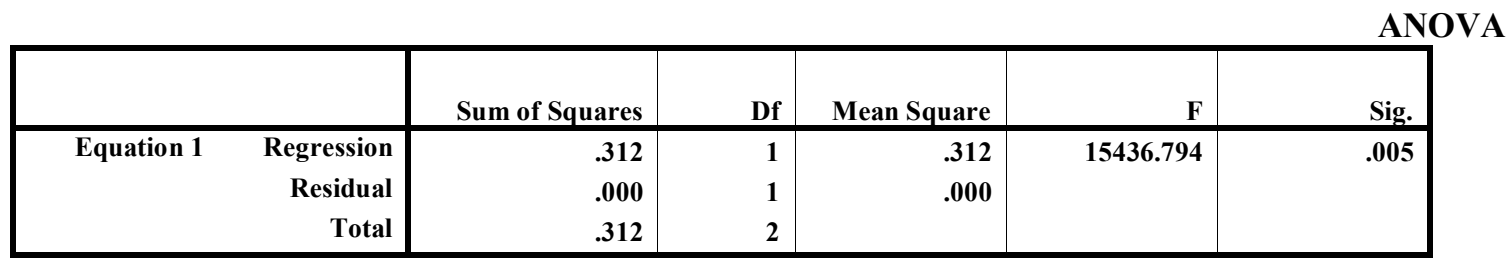

Coefficients

\begin{tabular}{|c|c|c|c|c|c|c|}
\hline & & \multicolumn{2}{|c|}{ Unstandardized Coefficients } & \multirow[t]{2}{*}{ Beta } & \multirow[t]{2}{*}{$\mathbf{T}$} & \multirow[t]{2}{*}{ Sig. } \\
\hline & & B & Std. Error & & & \\
\hline \multirow[t]{2}{*}{ Equation 1} & (Constant) & -.005 & .006 & & -.830 & .559 \\
\hline & NoOfPatients & .003 & .000 & 1.000 & 124.245 & .005 \\
\hline
\end{tabular}

Graph:(4-1)- The chart below demonstrate the number of patients presented for thyroid gland ultrasound with different sex through this study in The Kingdom of Saudi Arabia (Eastern area) August 2007 to May 2009.

\section{Patients Sexes}

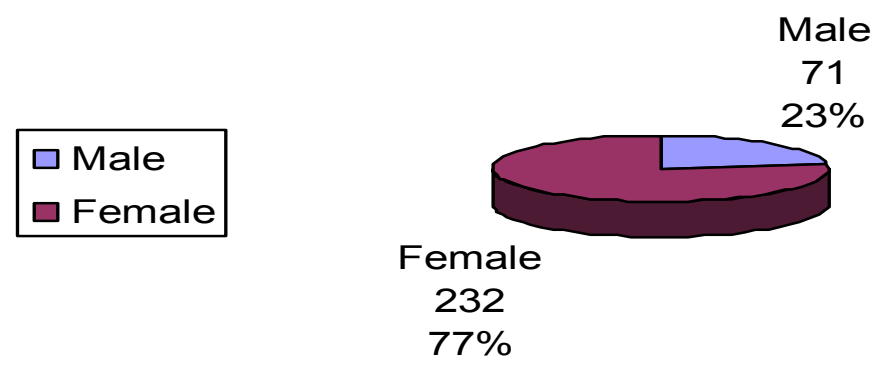

Table (4-2): Distribution of age groups among 303 patients scanned in ultrasound centers through this study in The Kingdom of Saudi Arabia (Eastern area) August 2007 to May 2009.

\begin{tabular}{|l|l|l|}
\hline Age groups & Number of patients & Percentage \\
\hline$(0-10)$ years & 6 & $2 \%$ \\
\hline$(10-20)$ years & 23 & $8 \%$ \\
\hline$(20-30)$ years & 48 & $16 \%$ \\
\hline$(30-40)$ years & 82 & $27 \%$ \\
\hline$(40-50)$ Years & 84 & $27 \%$ \\
\hline$(50-60)$ Years & 37 & $12 \%$ \\
\hline$(60-70)$ Years & 16 & $5 \%$ \\
\hline$(70-80)$ Years & 5 & $2 \%$ \\
\hline$(80-90)$ Years & 2 & $1 \%$ \\
\hline Total & 303 & $100 \%$ \\
\hline
\end{tabular}

Summarize

Case Processing Summary(a)

\begin{tabular}{|r|r|r|r|r|r|r|}
\hline & \multicolumn{9}{|c|}{ Cases } \\
\cline { 2 - 8 } & \multicolumn{3}{|c|}{ Included } & \multicolumn{1}{|c|}{ Excluded } & Total \\
\cline { 2 - 8 } & $\mathrm{N}$ & Percent & $\mathrm{N}$ & Percent & Percent \\
\hline NoOfPatients & 10 & $100.0 \%$ & 0 & $.0 \%$ & 10 & $100.0 \%$ \\
Percentage & 10 & $100.0 \%$ & 0 & $.0 \%$ & 10 & $100.0 \%$ \\
\hline
\end{tabular}

a Limited to first 100 cases. 


\begin{tabular}{|c|c|c|}
\hline & NoOfPatients & Percentage \\
\hline 1 & 6.00 & .02 \\
\hline 2 & 23.00 & .08 \\
\hline 3 & 48.00 & .16 \\
\hline 4 & 82.00 & .27 \\
\hline 5 & 84.00 & .27 \\
\hline 6 & 37.00 & .12 \\
\hline 7 & 16.00 & .05 \\
\hline 8 & 5.00 & .02 \\
\hline 9 & 2.00 & .01 \\
\hline 10 & 303.00 & 1.00 \\
\hline Total & 10 & 10 \\
\hline Mean & 60.6000 & .2000 \\
\hline Std. Deviation & 90.30098 & .29732 \\
\hline Range & 301.00 & .99 \\
\hline Std. Error of Skewness & .687 & .687 \\
\hline
\end{tabular}

Case Summaries(a)

a Limited to first 100 cases.

Descriptive Statistics

Descriptive

\begin{tabular}{|r|r|r|r|r|r|}
\hline & $\mathrm{N}$ & Minimum & Maximum & Mean & Std. Deviation \\
\hline NoOfPatients & 10 & 2.00 & 303.00 & 60.6000 & 90.30098 \\
Percentage & 10 & .01 & 1.00 & .2000 & .29732 \\
Valid N (listwise) & 10 & & & & \\
\hline
\end{tabular}

One-Sample Statistics

T-Test

\begin{tabular}{|r|r|r|r|r|}
\hline & $\mathrm{N}$ & Mean & Std. Deviation & $\begin{array}{r}\text { Std. Error } \\
\text { Mean }\end{array}$ \\
\hline NoOfPatients & 10 & 60.6000 & 90.30098 & 28.55568 \\
Percentage & 10 & .2000 & .29732 & .09402 \\
\hline
\end{tabular}

One-Sample Test

\begin{tabular}{|c|c|c|c|c|c|c|}
\hline & \multicolumn{6}{|c|}{ Test Value $=0$} \\
\hline & \multirow[b]{2}{*}{$\mathbf{t}$} & \multirow[b]{2}{*}{ df } & \multirow[b]{2}{*}{ Sig. (2-tailed) } & \multirow[b]{2}{*}{$\begin{array}{r}\text { Mean } \\
\text { Difference } \\
\end{array}$} & \multicolumn{2}{|c|}{$\begin{array}{r}\text { 95\% Confidence Interval of } \\
\text { the Difference }\end{array}$} \\
\hline & & & & & Lower & Upper \\
\hline NoOfPatients & 2.122 & 9 & .063 & 60.60000 & -3.9974 & 125.1974 \\
\hline Percentage & 2.127 & 9 & .062 & .20000 & -.0127 & .4127 \\
\hline
\end{tabular}

Two-stage Least Squares Analysis

Model Description

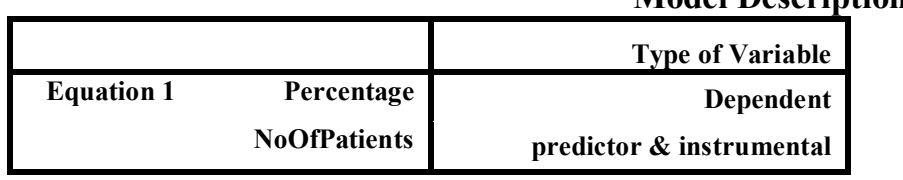




\begin{tabular}{|r|r|}
\hline Multiple R & Model Summary \\
R Square & 1.000 \\
Adjusted R Square & 1.000 \\
& 1.000 \\
Std. Error of the Estimate & .004 \\
\hline
\end{tabular}

\begin{tabular}{|rr|r|r|r|r|r|}
\multicolumn{10}{|c|}{ ANOVA } \\
\hline & & & & & \\
Segression & Sum of Squares & df & Mean Square & F & Sig. \\
\hline Equation 1 & Residual & .795 & 1 & .795 & 61561.086 & \\
& Total & .000 & 8 & .000 & & \\
& .796 & 9 & & & \\
\hline
\end{tabular}

\begin{tabular}{|rr|r|r|r|r|r|}
\hline & & & & & & \\
& & & Unstandardized \\
& & Coefficients & Beta & T & \\
\hline & B & Std. Error & & & \\
\hline Equation 1 & (Constant) & .000 & .001 & & .347 & .737 \\
& NoOfPatients & .003 & .000 & 1.000 & 248.115 & .000 \\
\hline
\end{tabular}

Graph:(4-2)- The chart below demonstrate the distribution of age groups among 303 patients scanned in ultrasound centers through this study in The Kingdom of Saudi Arabia (Eastern area) August 2007 to May 2009.

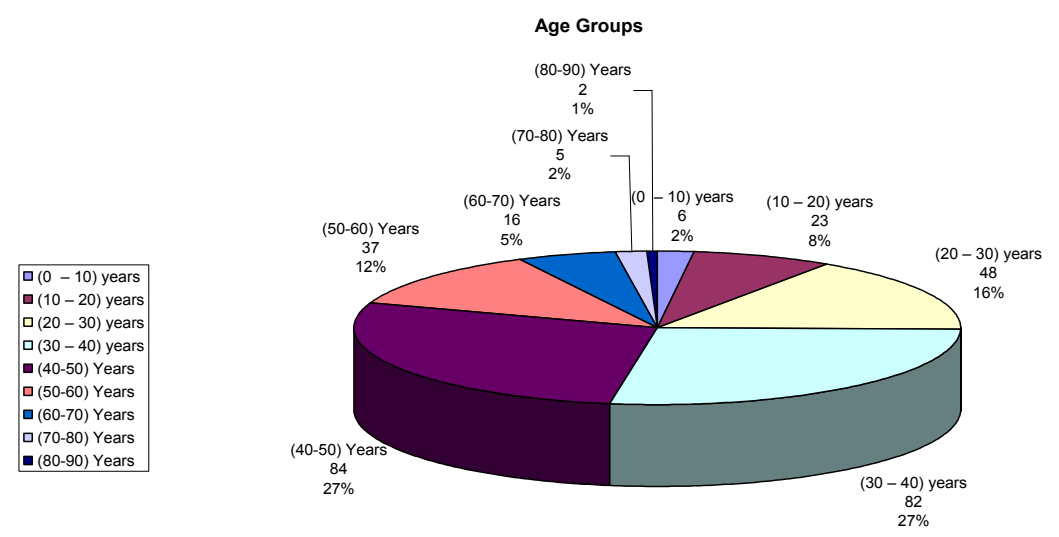

Table (4-3): Symptoms and signs in 303 patients investigated in ultrasound centers through this study in the Kingdom of Saudi Arabia (Eastern area) August 2007 to May 2009.

\begin{tabular}{|r|r|r|}
\hline Patient condition & Number of patients & Percentage \\
\hline Symptomatic & 224 & $74 \%$ \\
\hline A symptomatic & 79 & $26 \%$ \\
\hline Total & 303 & $100 \%$ \\
\hline
\end{tabular}

Case Processing Summary(a)

Summarize

\begin{tabular}{|r|r|r|r|r|r|r|}
\hline & \multicolumn{9}{|c|}{ Cases } \\
\cline { 2 - 7 } & \multicolumn{3}{|c|}{ Included } & \multicolumn{1}{c}{ Excluded } & Total \\
\cline { 2 - 7 } & $\mathrm{N}$ & Percent & $\mathrm{N}$ & Percent & $\mathrm{N}$ & Percent \\
\hline NoOfPatients & 3 & $100.0 \%$ & 0 & $.0 \%$ & 3 & $100.0 \%$ \\
Percentage & 3 & $100.0 \%$ & 0 & $.0 \%$ & 3 & $100.0 \%$ \\
\hline
\end{tabular}

a Limited to first 100 cases. 


\begin{tabular}{|c|c|c|}
\hline & NoOfPatients & Percentage \\
\hline 1 & 224.00 & .74 \\
\hline 2 & 79.00 & .26 \\
\hline 3 & 303.00 & 1.00 \\
\hline Total & 3 & 3 \\
\hline Mean & 202.0000 & .6667 \\
\hline Std. Deviation & 113.60898 & .37541 \\
\hline Range & 224.00 & .74 \\
\hline Std. Error of Skewness & 1.225 & 1.225 \\
\hline
\end{tabular}

Case Summaries(a)

a Limited to first 100 cases.

Descriptive Statistics

Descriptive

\begin{tabular}{|r|r|r|r|r|r|}
\hline & $\mathrm{N}$ & Minimum & Maximum & Mean & Std. Deviation \\
\hline NoOfPatients & 3 & 79.00 & 303.00 & 202.0000 & 113.60898 \\
Percentage & 3 & .26 & 1.00 & .6667 & .37541 \\
Valid N (listwise) & 3 & & & & \\
\hline
\end{tabular}

One-Sample Statistics

T-Test

\begin{tabular}{|r|r|r|r|r|}
\hline & & & & Std. Error \\
& $\mathrm{N}$ & Mean & Std. Deviation & Mean \\
\hline NoOfPatients & 3 & 202.0000 & 113.60898 & 65.59217 \\
Percentage & 3 & .6667 & .37541 & .21674 \\
\hline
\end{tabular}

One-Sample Test

\begin{tabular}{|c|c|c|c|c|c|c|}
\hline & \multicolumn{6}{|c|}{ Test Value $=0$} \\
\hline & \multirow[b]{2}{*}{$\mathbf{t}$} & \multirow[b]{2}{*}{ df } & \multirow[b]{2}{*}{ Sig. (2-tailed) } & \multirow{2}{*}{$\begin{array}{r}\text { Mean } \\
\text { Difference } \\
\end{array}$} & \multicolumn{2}{|c|}{$\begin{array}{r}\text { 95\% Confidence Interval of } \\
\text { the Difference }\end{array}$} \\
\hline & & & & & Lower & Upper \\
\hline NoOfPatients & 3.080 & 2 & .091 & 202.00000 & -80.2203 & 484.2203 \\
\hline Percentage & 3.076 & 2 & .091 & .66667 & -.2659 & 1.5992 \\
\hline
\end{tabular}

Two-stage Least Squares Analysis Model Description

\begin{tabular}{|rr|r|}
\hline & Type of Variable \\
\hline Equation 1 & Percentage & Dependent \\
& NoOfPatients & predictor \& instrumental \\
\hline
\end{tabular}

MOD_10

Model Summary

\begin{tabular}{|c|c|c|}
\hline Equation 1 & $\begin{array}{r}\text { Multiple R } \\
\text { R Square } \\
\text { Adjusted R Square } \\
\text { Std. Error of the Estimate }\end{array}$ & $\begin{array}{r}1.000 \\
1.000 \\
1.000 \\
.001\end{array}$ \\
\hline
\end{tabular}


ANOVA

\begin{tabular}{|rr|r|r|r|r|r|}
\hline & & & & & F \\
\hline Equation 1 & Sum of Squares & df & Mean Square & Sig. \\
& Ressidual & .282 & 1 & .282 & 450997.314 & .001 \\
& Total & .000 & 1 & .000 & & \\
\hline
\end{tabular}

\section{Coefficients}

\begin{tabular}{|rr|r|r|r|r|r|}
\hline & & Unstandardized Coefficients & Beta & S & Sig. \\
\hline & & B & Std. Error & & & \\
\hline Equation 1 & (Constant) & -.001 & .001 & & -.753 & .589 \\
& NoOfPatients & .003 & .000 & 1.000 & 671.563 & .001 \\
\hline
\end{tabular}

Graph:(4-3)- The chart below demonstrate the signs in 303 patients investigated in ultrasound centers through this study in the Kingdom of Saudi Arabia (Eastern area) August 2007 to May 2009.

\section{Symptomatic and a symptomatic patients}

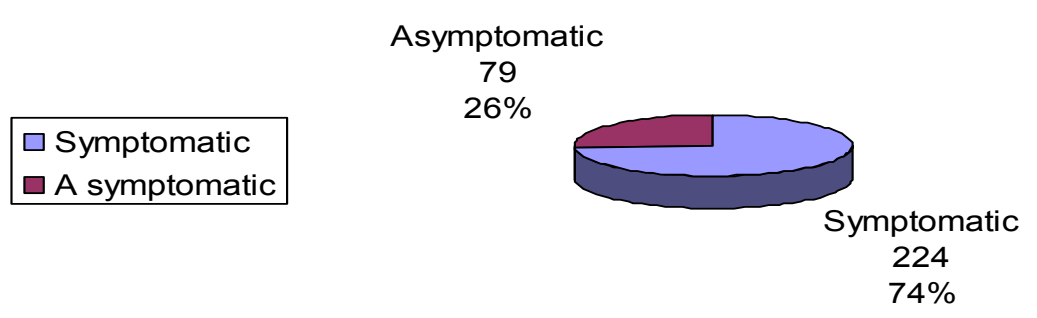

Table (4-4): Different signs and symptoms through (224 Symptomatic patients) scanned in ultrasound departments through this study in the kingdom of Saudi Arabia (Eastern area) August 2007 to April

2009.

\begin{tabular}{|c|c|c|}
\hline Symptoms & Number of patients & Percentage \\
\hline Pain & 75 & $32 \%$ \\
\hline Tenderness & 11 & $5 \%$ \\
\hline Compression of the respiratory tract & 7 & $3 \%$ \\
\hline $\begin{array}{l}\text { Problems with swallowing or inappropriate fixation } \\
\text { of the neck }\end{array}$ & 11 & $5 \%$ \\
\hline Vocal fold paralysis & 2 & $1 \%$ \\
\hline Neck swelling & 35 & $15 \%$ \\
\hline Hyperthyroidism & 45 & $19 \%$ \\
\hline Hypothyroidism & 10 & $4 \%$ \\
\hline Goiter & 28 & $12 \%$ \\
\hline Enlarge thyroid & 1 & $\mathbf{0} \%$ \\
\hline Thyroid nodule & 9 & $4 \%$ \\
\hline Sweating & 1 & $\mathbf{0} \%$ \\
\hline Nervousness and anxiety & 1 & $0 \%$ \\
\hline Fullness of neck & 1 & $0 \%$ \\
\hline Thyroiditis & 1 & $0 \%$ \\
\hline Following tiny nodule & 1 & $\mathbf{0 \%}$ \\
\hline Adenomyopathy elevated T3, T4 and TSH & 1 & $0 \%$ \\
\hline Graves's disease & 1 & $0 \%$ \\
\hline Total & 241 & $100 \%$ \\
\hline
\end{tabular}


Summarize

Case Processing Summary(a)

\begin{tabular}{|c|c|c|c|c|c|c|}
\hline & \multicolumn{6}{|r|}{ Cases } \\
\hline & \multicolumn{2}{|r|}{ Included } & \multicolumn{2}{|r|}{ Excluded } & \multicolumn{2}{|r|}{ Total } \\
\hline & $\mathbf{N}$ & Percent & $\mathbf{N}$ & Percent & $\mathbf{N}$ & Percent \\
\hline NoOfPatients & 19 & $100.0 \%$ & $\mathbf{0}$ & $.0 \%$ & 19 & $100.0 \%$ \\
\hline Percentage & 19 & $100.0 \%$ & $\mathbf{0}$ & $.0 \%$ & 19 & $100.0 \%$ \\
\hline
\end{tabular}

a Limited to first 100 cases.

\section{Case Summaries(a)}

\begin{tabular}{|c|c|c|c|}
\hline & & NoOfPatients & Percentage \\
\hline & 1 & 75.00 & .32 \\
\hline & 2 & 11.00 & .05 \\
\hline & 3 & 7.00 & .03 \\
\hline & 4 & 11.00 & .05 \\
\hline & 5 & 2.00 & .01 \\
\hline & 6 & 35.00 & .15 \\
\hline & 7 & 45.00 & .19 \\
\hline & 8 & 10.00 & .04 \\
\hline & 9 & 28.00 & .12 \\
\hline & 10 & 1.00 & .00 \\
\hline & 11 & 9.00 & .04 \\
\hline & 12 & 1.00 & .00 \\
\hline & 13 & 1.00 & .00 \\
\hline & 14 & 1.00 & .00 \\
\hline & 15 & 1.00 & .00 \\
\hline & 16 & 1.00 & .00 \\
\hline & 17 & 1.00 & .00 \\
\hline & 18 & 1.00 & .00 \\
\hline & 19 & 241.00 & 1.00 \\
\hline Total & $\mathbf{N}$ & 19 & 19 \\
\hline & Mean & 25.3684 & .1053 \\
\hline & Std. Deviation & 55.77555 & .23263 \\
\hline & Range & 240.00 & 1.00 \\
\hline & Std. Error of Skewness & .524 & .524 \\
\hline
\end{tabular}

a Limited to first 100 cases.

Descriptive Statistics

Descriptive

\begin{tabular}{|r|r|r|r|r|r|}
\hline & $\mathrm{N}$ & Minimum & Maximum & Mean & Std. Deviation \\
\hline NoOfPatients & 19 & 1.00 & 241.00 & 25.3684 & $\mathbf{5 5 . 7 7 5 5 5}$ \\
Percentage & 19 & .00 & 1.00 & .1053 & .23263 \\
Valid N (listwise) & 19 & & & & \\
\hline
\end{tabular}

T-Test 
One-Sample Statistics

\begin{tabular}{|r|r|r|r|r|}
\hline & $\mathrm{N}$ & Mean & Std. Deviation & $\begin{array}{r}\text { Std. Error } \\
\text { Mean }\end{array}$ \\
\hline NoOfPatients & 19 & 25.3684 & 55.77555 & 12.79579 \\
Percentage & 19 & .1053 & .23263 & .05337 \\
\hline
\end{tabular}

One-Sample Test

\begin{tabular}{|c|c|c|c|c|c|c|}
\hline & \multicolumn{6}{|c|}{ Test Value $=0$} \\
\hline & \multirow[b]{2}{*}{$\mathbf{t}$} & \multirow[b]{2}{*}{ df } & \multirow[b]{2}{*}{ Sig. (2-tailed) } & \multirow{2}{*}{$\begin{array}{r}\text { Mean } \\
\text { Difference }\end{array}$} & \multicolumn{2}{|c|}{$\begin{array}{r}95 \% \text { Confidence Interval of } \\
\text { the Difference }\end{array}$} \\
\hline & & & & & Lower & Upper \\
\hline NoOfPatients & 1.983 & 18 & .063 & 25.36842 & -1.5145 & $\mathbf{5 2 . 2 5 1 4}$ \\
\hline Percentage & 1.972 & 18 & .064 & .10526 & -.0069 & .2174 \\
\hline
\end{tabular}

Two-stage Least Squares Analysis

Model Description

\begin{tabular}{|rr|r|}
\hline & Type of Variable \\
\hline Equation 1 & Percentage & dependent \\
& NoOfPatients & predictor \& instrumental \\
\hline
\end{tabular}

Model Summary

MOD_11

\begin{tabular}{|r|r|}
\hline Equation 1 & Multiple R \\
R Square & 1.000 \\
Adjusted R Square & 1.000 \\
Std. Error of the Estimate & 1.000 \\
& .004 \\
\hline
\end{tabular}

ANOVA

\begin{tabular}{|rr|r|r|r|r|r|}
\hline & $\begin{array}{r}\text { Sum of } \\
\text { Squares }\end{array}$ & df & Mean Square & Sig. \\
\hline Equation 1 & Regression & .974 & 1 & .974 & 57061.779 & .000 \\
& Residual & .000 & 17 & .000 & \\
& Total & .974 & 18 & & \\
\end{tabular}

Coefficients

\begin{tabular}{|rr|r|r|r|r|r|}
\hline & \multicolumn{2}{|c|}{$\begin{array}{r}\text { Unstandardized } \\
\text { Coefficients }\end{array}$} & Beta & t & \\
\hline & & B & Std. Error & & & \\
\hline Equation 1 & (Constant) & -.001 & .001 & & -.504 & .621 \\
& NoOfPatients & .004 & .000 & $\mathbf{1 . 0 0 0}$ & $\mathbf{2 3 8 . 8 7 6}$ & .000 \\
\hline
\end{tabular}

Graph:(4-4)- The next chart demonstrate the different signs and symptoms through (224 Symptomatic patients) scanned in ultrasound departments through this study in the kingdom of Saudi Arabia (Eastern area) August 2007 to May 2009. 


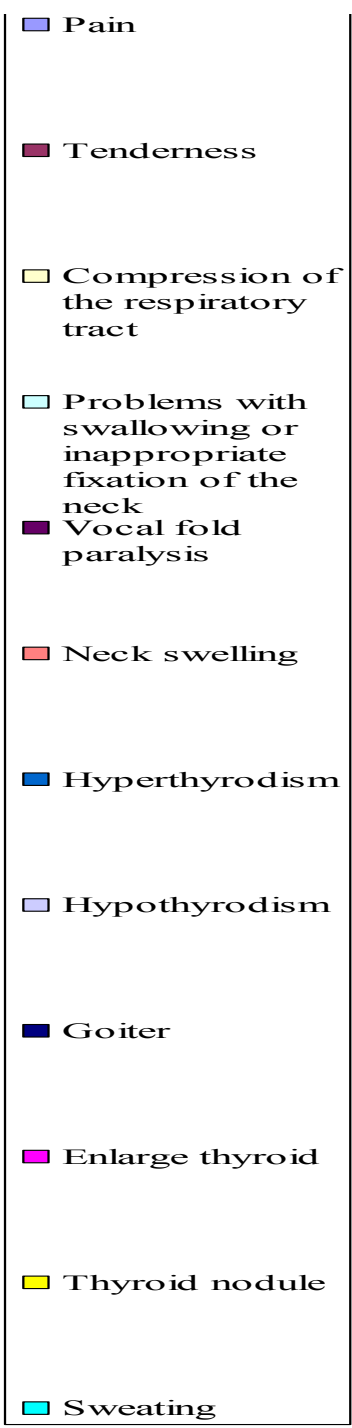

Different Symptoms

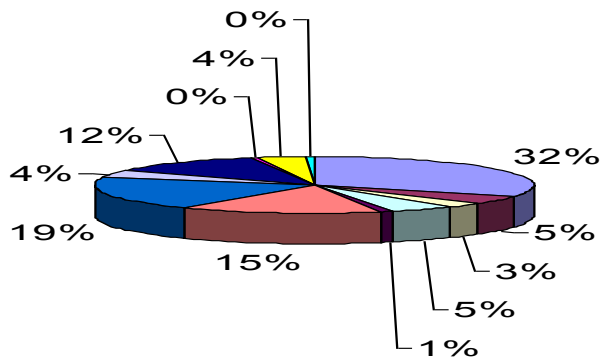

Table (4-5): Ultrasound findings through 303 patients scanned in ultrasound departments through this study in the kingdom of Saudi Arabia (Eastern area) August 2007 to May 2009.

\begin{tabular}{|c|c|c|}
\hline Ultrasound findings & Number of patients & Percentage \\
\hline Thyroid enlargement & 147 & $25 \%$ \\
\hline Solitary thyroid nodules & 131 & $23 \%$ \\
\hline Multinodular goiters & 55 & $10 \%$ \\
\hline Cyst & 45 & $8 \%$ \\
\hline Nonpalpable thyroid nodules & 42 & $7 \%$ \\
\hline Goiter & 23 & $4 \%$ \\
\hline FNAC & 23 & $4 \%$ \\
\hline Hashimoto's thyroiditis (HT) & 18 & $3 \%$ \\
\hline A solid hypoechoic appearance & 14 & $2 \%$ \\
\hline Nodal enlargement & 12 & $2 \%$ \\
\hline Adenoma & 11 & $2 \%$ \\
\hline Increase vascularity & 9 & $2 \%$ \\
\hline Average size of gland & 9 & $2 \%$ \\
\hline Thyroiditis & 7 & $2 \%$ \\
\hline Microcalcifications & 6 & $1 \%$ \\
\hline Small size thyroid & 6 & $1 \%$ \\
\hline Intranodular vascular pattern & 5 & $1 \%$ \\
\hline Multiple thyroid nodules & 5 & $1 \%$ \\
\hline Thyroglossal cyst & 4 & $1 \%$ \\
\hline Graves disease & 3 & $1 \%$ \\
\hline Irregular or blurred margins & 2 & $0 \%$ \\
\hline Swelling & 2 & 0\% \\
\hline
\end{tabular}


Role Of Ultrasound In Thyroid Pathologies

\begin{tabular}{|c|c|c|}
\hline Mediastinum extension & 1 & $0 \%$ \\
\hline Intranodular vascular spots & 1 & $0 \%$ \\
\hline Isoechoic area & 1 & $0 \%$ \\
\hline Hypovascularity & 1 & $0 \%$ \\
\hline Complex mass & 1 & $0 \%$ \\
\hline Lipoma & 1 & $0 \%$ \\
\hline Hyperplasia & 1 & $0 \%$ \\
\hline Hyperthyroidism & 1 & $0 \%$ \\
\hline
\end{tabular}

Summarize

Case Processing Summary(a)

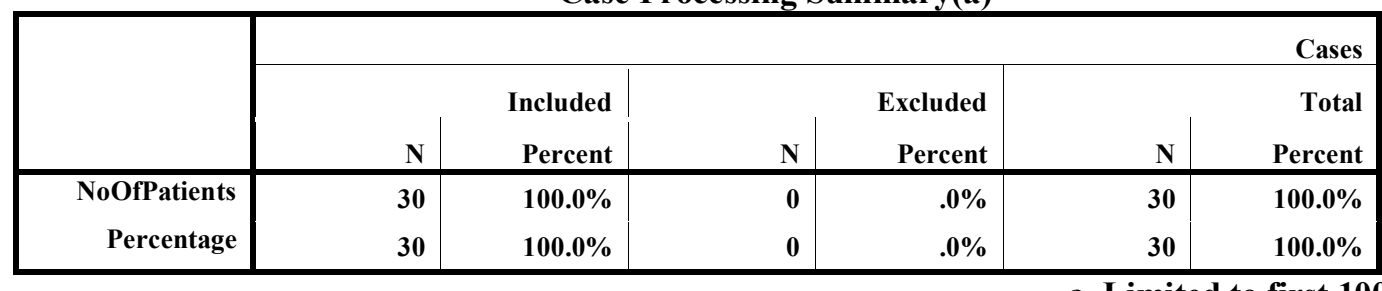

a Limited to first 100 cases.

\begin{tabular}{|c|c|c|c|}
\hline & & NoOfPatients & Percentage \\
\hline & 1 & 147.00 & .25 \\
\hline & 2 & 131.00 & .23 \\
\hline & 3 & 55.00 & .01 \\
\hline & 4 & 45.00 & .08 \\
\hline & 5 & 42.00 & .07 \\
\hline & 6 & 23.00 & .04 \\
\hline & 7 & 23.00 & .04 \\
\hline & 8 & 18.00 & .03 \\
\hline & 9 & 14.00 & .02 \\
\hline & 10 & 12.00 & .02 \\
\hline & 11 & 11.00 & .02 \\
\hline & 12 & 9.00 & .02 \\
\hline & 13 & 9.00 & .02 \\
\hline & 14 & 7.00 & .02 \\
\hline & 15 & 6.00 & .01 \\
\hline & 16 & 6.00 & .01 \\
\hline & 17 & 5.00 & .01 \\
\hline & 18 & 5.00 & .01 \\
\hline & 19 & 4.00 & .01 \\
\hline & 20 & 3.00 & .01 \\
\hline & 21 & 2.00 & .00 \\
\hline & 22 & 2.00 & .00 \\
\hline & 23 & 1.00 & .00 \\
\hline & 24 & 1.00 & .00 \\
\hline & 25 & 1.00 & .00 \\
\hline & 26 & 1.00 & .00 \\
\hline & 27 & 1.00 & .00 \\
\hline & 28 & 1.00 & .00 \\
\hline & 29 & 1.00 & .00 \\
\hline & 30 & 1.00 & .00 \\
\hline Total & $\mathbf{N}$ & 30 & 30 \\
\hline & Mean & 19.5667 & .0310 \\
\hline & Std. Deviation & 35.38720 & .06019 \\
\hline & Range & 146.00 & .25 \\
\hline & Std. Error of Skewness & .427 & .427 \\
\hline
\end{tabular}

Case Summaries(a)

a Limited to first 100 cases. 
Descriptive Statistics

\begin{tabular}{|r|r|r|r|r|r|}
\hline & $\mathrm{N}$ & Minimum & Maximum & Mean & Std. Deviation \\
\hline NoOfPatients & 30 & 1.00 & 147.00 & 19.5667 & 35.38720 \\
Percentage & 30 & .00 & .25 & .0310 & .06019 \\
Valid N (listwise) & 30 & & & & \\
\hline
\end{tabular}

One-Sample Statistics

T-Test

\begin{tabular}{|r|r|r|r|r|}
\hline & $\mathrm{N}$ & Mean & Std. Deviation & $\begin{array}{r}\text { Std. Error } \\
\text { Mean }\end{array}$ \\
\hline NoOfPatients & 30 & 19.5667 & $\mathbf{3 5 . 3 8 7 2 0}$ & 6.46079 \\
Percentage & 30 & .0310 & .06019 & .01099 \\
\hline
\end{tabular}

One-Sample Test

\begin{tabular}{|c|c|c|c|c|c|c|}
\hline & \multicolumn{6}{|c|}{ Test Value $=0$} \\
\hline & \multirow[b]{2}{*}{$\mathbf{t}$} & \multirow[b]{2}{*}{ df } & \multirow[b]{2}{*}{ Sig. (2-tailed) } & \multirow{2}{*}{$\begin{array}{r}\text { Mean } \\
\text { Difference }\end{array}$} & \multicolumn{2}{|c|}{$\begin{array}{r}95 \% \text { Confidence Interval of } \\
\text { the Difference }\end{array}$} \\
\hline & & & & & Lower & Upper \\
\hline NoOfPatients & 3.029 & 29 & .005 & 19.56667 & 6.3529 & 32.7805 \\
\hline Percentage & 2.821 & 29 & .009 & .03100 & .0085 & .0535 \\
\hline
\end{tabular}

Two-stage Least Squares Analysis

\begin{tabular}{|rr|r|}
\hline & Type of Variable \\
\hline Equation 1 & Percentage & dependent \\
& NoOfPatients & predictor \& instrumental \\
\hline
\end{tabular}

Model Description

\begin{tabular}{|r|r|}
\multicolumn{2}{c}{ Model Summary } \\
\hline Equation 1 & .966 \\
R Square & .934 \\
Adjusted R Square & .931 \\
Std. Error of the Estimate & .016 \\
\hline
\end{tabular}

MOD_12

ANOVA

\begin{tabular}{|rr|r|r|r|r|r|}
\hline & & & & \\
& & Df & Mean Square & F & Sig. \\
\hline Equation 1 & Regression & .098 & 1 & .098 & 393.295 \\
& Residual & .007 & 28 & .000 & \\
& Total & .105 & 29 & & \\
\end{tabular}

Coefficients

\begin{tabular}{|rr|r|r|r|r|r|}
\hline & & \multicolumn{2}{|c|}{ Unstandardized Coefficients } & & \\
\cline { 3 - 7 } & & B & Std. Error & Beta & Sig. \\
\hline Equation 1 & (Constant) & -.001 & .003 &. .350 & t \\
& NoOfPatients & .002 & .000 & .966 & 19.832 & .000 \\
\hline
\end{tabular}

Graph:(4-5)- The next chart demonstrate the ultrasound findings through 303 patients scanned in ultrasound departments through this study in the kingdom of Saudi Arabia (Eastern area) August 2007 to May 2009. 

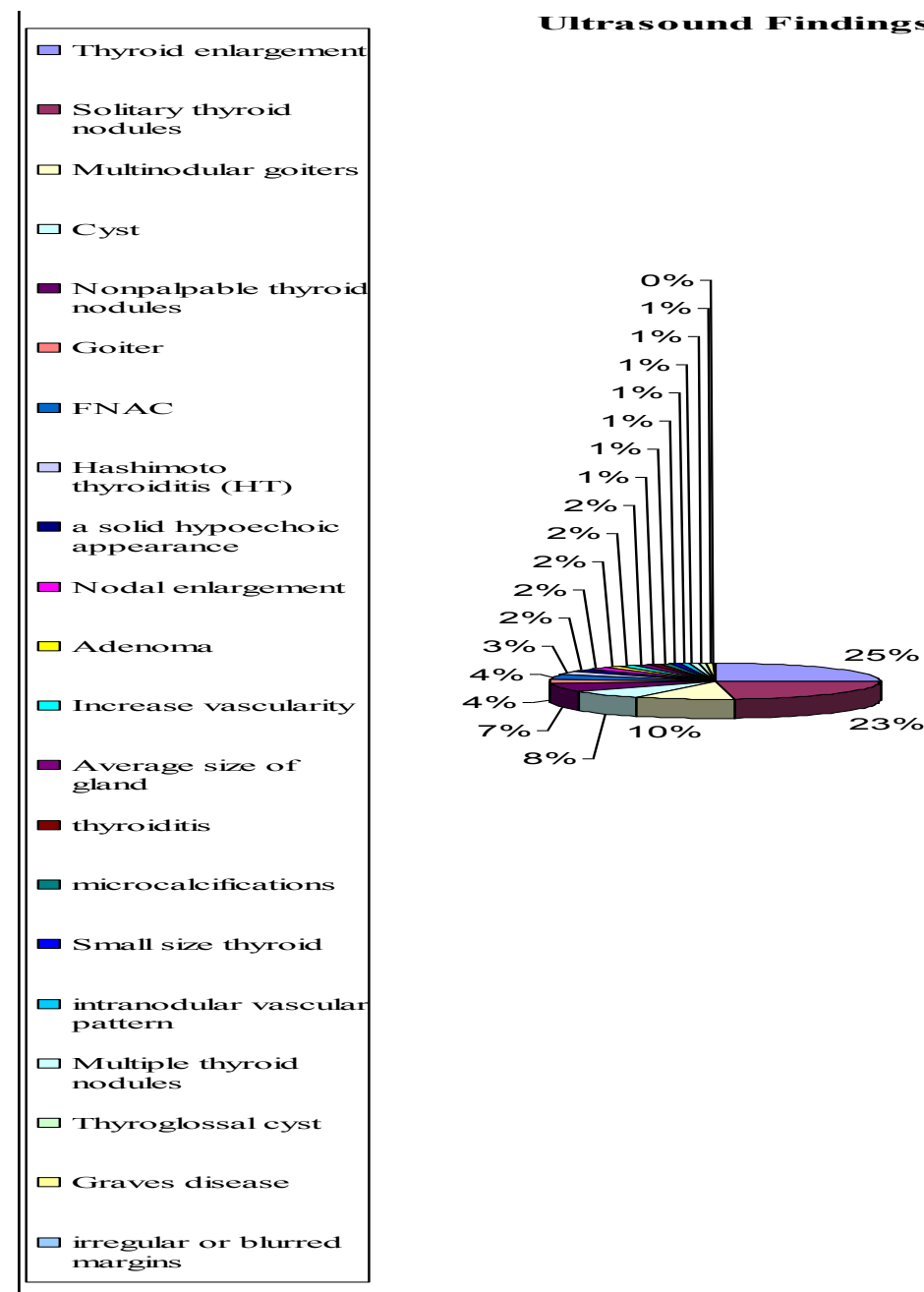

Table (4-6): Symptoms and signs in 172 symptomatic female patients investigated in ultrasound centers through this study in the Kingdom of Saudi Arabia (Eastern area) August 2007 to May 2009.

\begin{tabular}{|c|c|c|}
\hline Symptoms & Number of patients & Percentage \\
\hline Pain & 57 & $32 \%$ \\
\hline Hyperthyroidism & 36 & $20 \%$ \\
\hline Neck swelling & 22 & $12 \%$ \\
\hline Goiter & 22 & $12 \%$ \\
\hline $\begin{array}{l}\text { Problems with swallowing or inappropriate } \\
\text { fixation of the neck }\end{array}$ & 10 & $5 \%$ \\
\hline Tenderness & 9 & $5 \%$ \\
\hline Compression of the respiratory tract & 7 & $4 \%$ \\
\hline Hypothyroidism & 8 & $4 \%$ \\
\hline Thyroid nodule & 6 & $3 \%$ \\
\hline Vocal fold paralysis & 2 & $1 \%$ \\
\hline Thyrotoxic & 2 & $1 \%$ \\
\hline Enlarge thyroid & 1 & $0 \%$ \\
\hline Sweating & 1 & $0 \%$ \\
\hline Nervousness and anxiety & 1 & $0 \%$ \\
\hline Fullness of neck & 1 & $0 \%$ \\
\hline Thyroiditis & 1 & $0 \%$ \\
\hline Adenomyopathy elevated T3, T4 and TSH & 1 & $0 \%$ \\
\hline Graves's disease & 1 & $0 \%$ \\
\hline
\end{tabular}

Summarize 
Case Processing Summary(a)

\begin{tabular}{|c|c|c|c|c|c|c|}
\hline & \multicolumn{6}{|r|}{ Cases } \\
\hline & & Included & & Excluded & & Total \\
\hline & $\mathbf{N}$ & Percent & $\mathbf{N}$ & Percent & $\mathbf{N}$ & Percent \\
\hline NoOfPatients & 18 & $100.0 \%$ & $\mathbf{0}$ & $.0 \%$ & 18 & $100.0 \%$ \\
\hline Percentage & 18 & $100.0 \%$ & $\mathbf{0}$ & $.0 \%$ & 18 & $100.0 \%$ \\
\hline
\end{tabular}

a Limited to first 100 cases.

Case Summaries(a)

\begin{tabular}{|c|c|c|}
\hline & NoOfPatients & Percentage \\
\hline 1 & 57.00 & .32 \\
\hline 2 & 36.00 & .20 \\
\hline 3 & 22.00 & .12 \\
\hline 4 & 22.00 & .12 \\
\hline 5 & 10.00 & .05 \\
\hline 6 & 9.00 & .05 \\
\hline 7 & 7.00 & .04 \\
\hline 8 & 8.00 & .04 \\
\hline 9 & 6.00 & .03 \\
\hline 10 & 2.00 & .01 \\
\hline 11 & 2.00 & .01 \\
\hline 12 & 1.00 & .00 \\
\hline 13 & 1.00 & .00 \\
\hline 14 & 1.00 & .00 \\
\hline 15 & 1.00 & .00 \\
\hline 16 & 1.00 & .00 \\
\hline 17 & 1.00 & .00 \\
\hline 18 & 1.00 & .00 \\
\hline Total & 18 & 18 \\
\hline Mean & 10.4444 & .0550 \\
\hline Std. Deviation & 15.13555 & .08611 \\
\hline Range & 56.00 & .32 \\
\hline Std. Error of Skewness & .536 & .536 \\
\hline
\end{tabular}

a Limited to first 100 cases.

Descriptive Statistics

Descriptive

\begin{tabular}{|r|r|r|r|r|r|}
\hline & $\mathrm{N}$ & Minimum & Maximum & Mean & Std. Deviation \\
\hline NoOfPatients & 18 & 1.00 & 57.00 & 10.4444 & 15.13555 \\
Percentage & 18 & .00 & .32 & .0550 & .08611 \\
Valid N (listwise) & 18 & & & & \\
\hline
\end{tabular}

One-Sample Statistics

T-Test

\begin{tabular}{|r|r|r|r|r|}
\hline & $\mathrm{N}$ & Mean & Std. Deviation & $\begin{array}{r}\text { Std. Error } \\
\text { Mean }\end{array}$ \\
\hline NoOfPatients & 18 & 10.4444 & 15.13555 & 3.56748 \\
Percentage & 18 & .0550 & .08611 & .02030 \\
\hline
\end{tabular}


One-Sample Test

\begin{tabular}{|c|c|c|c|c|c|c|}
\hline & \multicolumn{6}{|c|}{ Test Value $=0$} \\
\hline & \multirow[b]{2}{*}{$\mathbf{t}$} & \multirow[b]{2}{*}{ df } & \multirow[b]{2}{*}{ Sig. (2-tailed) } & \multirow{2}{*}{$\begin{array}{r}\text { Mean } \\
\text { Difference } \\
\end{array}$} & \multicolumn{2}{|c|}{$\begin{array}{r}\text { 95\% Confidence Interval of } \\
\text { the Difference }\end{array}$} \\
\hline & & & & & Lower & Upper \\
\hline NoOfPatients & 2.928 & 17 & .009 & 10.44444 & 2.9177 & 17.9712 \\
\hline Percentage & 2.710 & 17 & .015 & .05500 & .0122 & .0978 \\
\hline
\end{tabular}

Two-stage Least Squares Analysis Model Description

\begin{tabular}{|rr|r|}
\hline & Type of Variable \\
\hline Equation 1 & Percentage & dependent \\
& NoOfPatients & predictor \& instrumental \\
\hline
\end{tabular}

MOD_13

Model Summary

\begin{tabular}{|r|r|}
\hline Equation 1 & Multiple R \\
R Square & 1.000 \\
Adjusted R Square & .999 \\
& .999 \\
Std. Error of the Estimate & .002 \\
\hline
\end{tabular}

ANOVA

\begin{tabular}{|c|c|c|c|c|c|c|}
\hline & & $\begin{array}{r}\text { Sum of } \\
\text { Squares }\end{array}$ & df & Mean Square & $\mathbf{F}$ & Sig. \\
\hline \multirow[t]{3}{*}{ Equation 1} & Regression & .126 & 1 & .126 & 28836.588 & .000 \\
\hline & Residual & .000 & 16 & .000 & & \\
\hline & Total & .126 & 17 & & & \\
\hline
\end{tabular}

Coefficients

\begin{tabular}{|c|c|c|c|c|c|c|}
\hline & & \multicolumn{2}{|c|}{ Unstandardized Coefficients } & \multirow[t]{2}{*}{ Beta } & \multirow[t]{2}{*}{$\mathbf{t}$} & \multirow[t]{2}{*}{ Sig. } \\
\hline & & B & Std. Error & & & \\
\hline \multirow[t]{2}{*}{ Equation 1} & (Constant) & -.004 & .001 & & -7.288 & .000 \\
\hline & NoOfPatients & .006 & .000 & 1.000 & 169.813 & .000 \\
\hline
\end{tabular}

Graph:(4-6)- The next chart demonstrate the different signs and symptoms through (172 Symptomatic female patients) scanned in ultrasound departments through this study in the kingdom of Saudi Arabia (Eastern area) August 2007 to May 2009. 


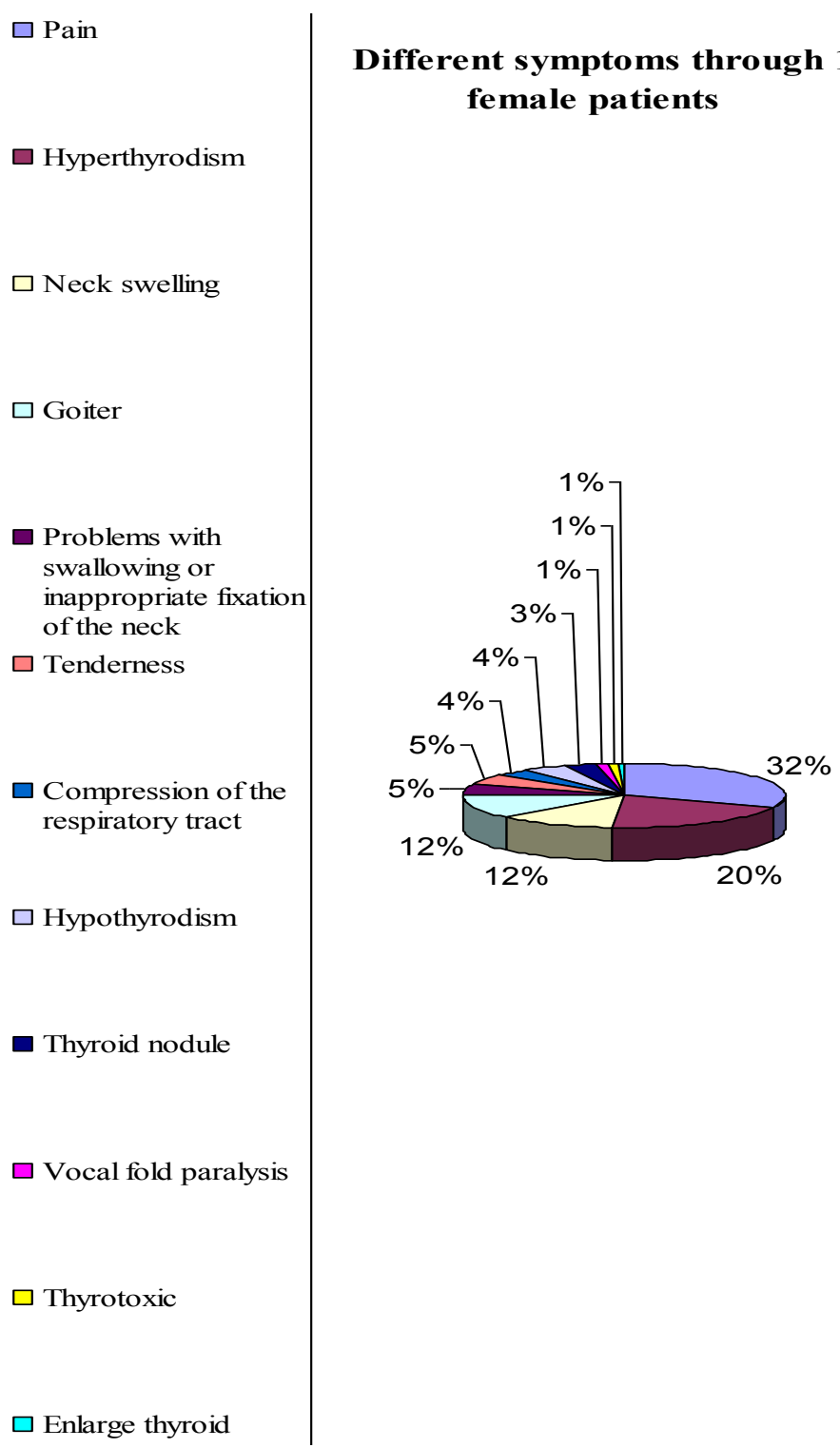

Table (4-7): Symptoms and signs in 52 symptomatic male patients investigated in ultrasound centers through this study in the Kingdom of Saudi Arabia (Eastern area) August 2007 to May 2009.

\begin{tabular}{|l|l|l|}
\hline Symptoms & Number of patients & Percentage \\
\hline Pain & 18 & $31 \%$ \\
\hline Neck swelling & 13 & $23 \%$ \\
\hline Hyperthyroidism & 9 & $16 \%$ \\
\hline Goiter & 6 & $11 \%$ \\
\hline Thyroid nodule & 4 & $7 \%$ \\
\hline Tenderness & 2 & $4 \%$ \\
\hline Hypothyroidism Post operative & 2 & $4 \%$ \\
\hline $\begin{array}{l}\text { Problems with swallowing or inappropriate } \\
\text { fixation of the neck }\end{array}$ & 1 & $2 \%$ \\
\hline & 56 & $2 \%$ \\
\hline Total & 2 & $100 \%$ \\
\hline
\end{tabular}

Summarize 
Case Processing Summary(a)

\begin{tabular}{|c|c|c|c|c|c|c|}
\hline & \multicolumn{6}{|r|}{ Cases } \\
\hline & \multicolumn{2}{|r|}{ Included } & \multicolumn{2}{|c|}{ Excluded } & \multicolumn{2}{|r|}{ Total } \\
\hline & $\mathbf{N}$ & Percent & $\mathbf{N}$ & Percent & $\mathbf{N}$ & Percent \\
\hline NoOfPatients & 10 & $100.0 \%$ & $\mathbf{0}$ & $.0 \%$ & 10 & $100.0 \%$ \\
\hline Percentahe & 10 & $100.0 \%$ & $\mathbf{0}$ & $.0 \%$ & 10 & $100.0 \%$ \\
\hline
\end{tabular}

a Limited to first 100 cases.

Case Summaries(a)

\begin{tabular}{|r|r|r|}
\hline & NoOfPatients & Percentahe \\
\hline 1 & 18.00 & .31 \\
2 & 13.00 & .23 \\
3 & 9.00 & .16 \\
4 & 6.00 & .11 \\
5 & 4.00 & .07 \\
6 & 2.00 & .04 \\
7 & 2.00 & .04 \\
8 & 1.00 & .02 \\
9 & 1.00 & .02 \\
10 & 56.00 & 1.00 \\
N & 10 & 10 \\
Mean & 11.2000 & .2000 \\
Std. Deviation & 16.72523 & .29732 \\
Range & 55.00 & .98 \\
Std. Error of Skewness & .687 & .687 \\
\hline
\end{tabular}

a Limited to first 100 cases.

Descriptive Statistics

Descriptives

\begin{tabular}{|r|r|r|r|r|r|}
\hline & $\mathrm{N}$ & Minimum & Maximum & Mean & Std. Deviation \\
\hline NoOfPatients & 10 & 1.00 & $\mathbf{5 6 . 0 0}$ & $\mathbf{1 1 . 2 0 0 0}$ & 16.72523 \\
Percentahe & 10 & .02 & 1.00 & .2000 & .29732 \\
Valid N (listwise) & 10 & & & & \\
\hline
\end{tabular}

One-Sample Statistics

T-Test

\begin{tabular}{|c|c|c|c|c|}
\hline & $\mathbf{N}$ & Mean & Std. Deviation & $\begin{array}{r}\text { Std. Error } \\
\text { Mean } \\
\end{array}$ \\
\hline NoOfPatients & 10 & 11.2000 & 16.72523 & 5.28898 \\
\hline Percentahe & 10 & .2000 & .29732 & .09402 \\
\hline
\end{tabular}

One-Sample Test

\begin{tabular}{|c|c|c|c|c|c|c|}
\hline & \multirow[b]{2}{*}{$\mathbf{t}$} & \multirow[b]{2}{*}{ df } & \multirow[b]{2}{*}{ Sig. (2-tailed) } & \multirow{2}{*}{$\begin{array}{r}\text { Mean } \\
\text { Difference }\end{array}$} & \multicolumn{2}{|c|}{$\begin{array}{r}\text { Test Value }=0 \\
\text { 95\% Confidence Interval of } \\
\text { the Difference }\end{array}$} \\
\hline & & & & & Lower & Upper \\
\hline $\begin{array}{r}\text { NoOfPatients } \\
\text { Percentahe }\end{array}$ & $\begin{array}{l}2.118 \\
2.127\end{array}$ & $\begin{array}{l}9 \\
9\end{array}$ & $\begin{array}{l}.063 \\
.062\end{array}$ & $\begin{array}{r}11.20000 \\
.20000\end{array}$ & $\begin{array}{l}-.7645 \\
-.0127\end{array}$ & $\begin{array}{r}23.1645 \\
.4127\end{array}$ \\
\hline
\end{tabular}


Two-stage Least Squares Analysis Model Description

\begin{tabular}{|rr|r|}
\hline & Type of Variable \\
\hline Equation 1 & Percentahe & dependent \\
& NoOfPatients & predictor \& instrumental \\
\hline
\end{tabular}

MOD_14

Model Summary

\begin{tabular}{|r|r|}
\hline Equation 1 & Multiple R \\
R Square & 1.000 \\
& 1.000 \\
Adjusted R Square & 1.000 \\
Std. Error of the Estimate & .005 \\
\hline
\end{tabular}

ANOVA

\begin{tabular}{|rr|r|r|r|r|r|}
\hline & $\begin{array}{r}\text { Sum of } \\
\text { Squares }\end{array}$ & df & Mean Square & F & Sig. \\
\hline Equation 1 & Regression & .795 & 1 & .795 & $\mathbf{3 6 4 0 5 . 2 6 3}$ & .000 \\
& Residual & .000 & $\mathbf{8}$ & .000 & & \\
& Total & .796 & 9 & & & \\
\hline
\end{tabular}

Coefficients

\begin{tabular}{|rr|r|r|r|r|r|}
\hline & & Unstandardized Coefficients & Beta & T & Sig. \\
\hline & & B & Std. Error & & & \\
\hline Equation 1 & (Constant) & .001 & .002 & & .509 & .624 \\
& NoOfPatient & .018 & .000 & 1.000 & 190.802 & .000 \\
\hline
\end{tabular}

Graph:(4-7)- The next chart demonstrate the different signs and symptoms through (52 Symptomatic male patients) scanned in ultrasound departments through this study in the kingdom of Saudi Arabia (Eastern area) August 2007 to May 2009.

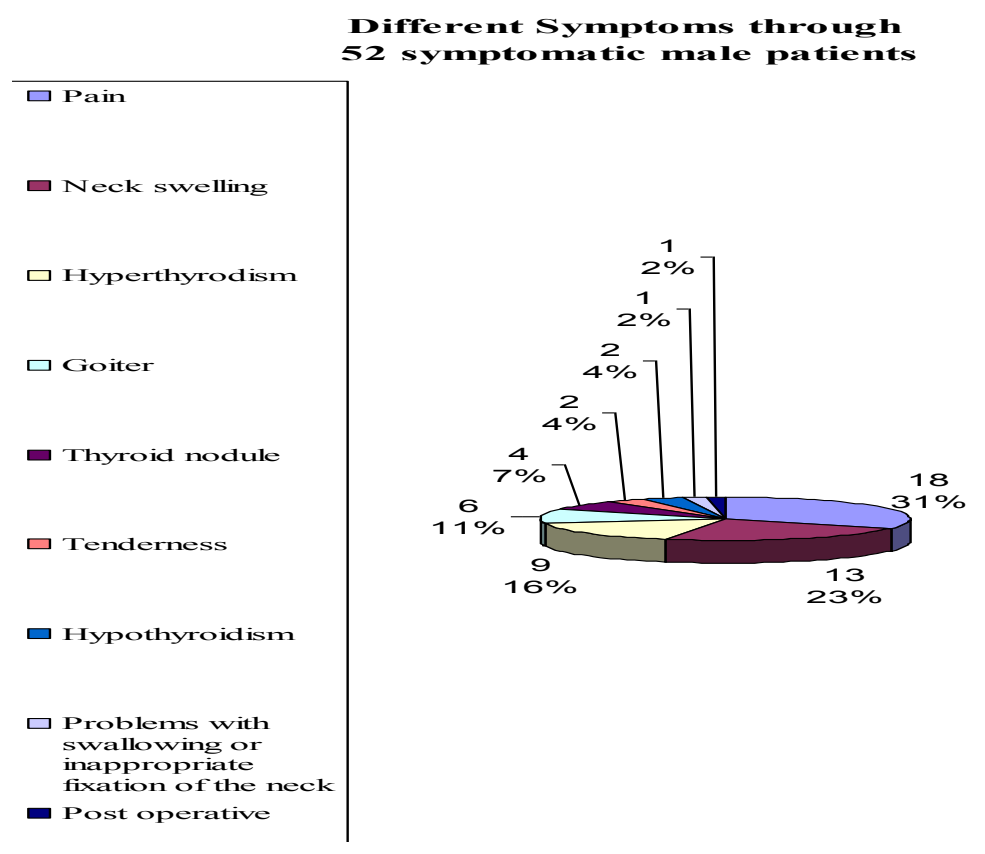


Table (4-8): Ultrasound findings through 232 female patients scanned in ultrasound departments through this study in the kingdom of Saudi Arabia (Eastern area) August 2007 to May 2009.

\begin{tabular}{|c|c|c|}
\hline Ultrasound finding & Number of patients & Percentage \\
\hline Thyroid enlargement & 110 & $24 \%$ \\
\hline Solitary thyroid nodules & 106 & $24 \%$ \\
\hline Multinodular goiters & 47 & $10 \%$ \\
\hline Cyst & 32 & $7 \%$ \\
\hline Nonpalpable thyroid nodules & 31 & $7 \%$ \\
\hline FNAC & 20 & $4 \%$ \\
\hline Hashimoto thyroiditis (HT) & 15 & $3 \%$ \\
\hline Goiter & 14 & $3 \%$ \\
\hline Nodal enlargement & 12 & $3 \%$ \\
\hline A solid hypoechoic appearance & 9 & $2 \%$ \\
\hline Increase vascularity & 9 & $2 \%$ \\
\hline Adenoma & 6 & $1 \%$ \\
\hline Thyroiditis & 6 & $1 \%$ \\
\hline Average size of gland & 5 & $1 \%$ \\
\hline Microcalcifications & 4 & $1 \%$ \\
\hline Small size thyroid & 4 & $1 \%$ \\
\hline Intranodular vascular pattern & 4 & $1 \%$ \\
\hline Multiple thyroid nodules & 3 & $1 \%$ \\
\hline Graves's disease & 3 & $1 \%$ \\
\hline Irregular or blurred margins & 2 & $0 \%$ \\
\hline Swelling & 2 & $0 \%$ \\
\hline Mediastinum extension & 1 & $0 \%$ \\
\hline Intranodular vascular spots & 1 & $0 \%$ \\
\hline Isoechoic area & 1 & $0 \%$ \\
\hline Hypovascularity & 1 & $0 \%$ \\
\hline Complex mass & 1 & $0 \%$ \\
\hline Thyroglossal cyst & 1 & $0 \%$ \\
\hline Hyperthyroidism & 1 & $0 \%$ \\
\hline
\end{tabular}

Case Processing Summary(a) Summarize

\begin{tabular}{|c|c|c|c|c|c|c|}
\hline & \multicolumn{6}{|r|}{ Cases } \\
\hline & \multicolumn{2}{|r|}{ Included } & \multicolumn{2}{|r|}{ Excluded } & \multicolumn{2}{|r|}{ Total } \\
\hline & $\mathbf{N}$ & Percent & $\mathbf{N}$ & Percent & $\mathbf{N}$ & Percent \\
\hline NoOfPatients & 28 & $100.0 \%$ & $\mathbf{0}$ & $.0 \%$ & 28 & $100.0 \%$ \\
\hline Percentage & 28 & $100.0 \%$ & $\mathbf{0}$ & $.0 \%$ & 28 & $100.0 \%$ \\
\hline
\end{tabular}

a Limited to first 100 cases.

Case Summaries(a)

\begin{tabular}{|r|r|r|}
\hline & NoOfPatients & Percentage \\
\hline 1 & 110.00 & .24 \\
2 & 106.00 & .24 \\
3 & 47.00 & .10 \\
4 & 32.00 & .07 \\
5 & 31.00 & .07 \\
6 & 20.00 & .04 \\
7 & 15.00 & .03 \\
8 & 14.00 & .03 \\
9 & 12.00 & .03 \\
10 & 9.00 & .02 \\
11 & 9.00 & .02 \\
12 & 6.00 & .01 \\
13 & 6.00 & .01 \\
14 & 5.00 & .01 \\
15 & 4.00 & .01 \\
16 & 4.00 & .01
\end{tabular}




\begin{tabular}{|c|c|c|c|}
\hline & 17 & 4.00 & .01 \\
\hline & 18 & 3.00 & .01 \\
\hline & 19 & 3.00 & .01 \\
\hline & 20 & 2.00 & .00 \\
\hline & 21 & 2.00 & .00 \\
\hline & 22 & 1.00 & .00 \\
\hline & 23 & 1.00 & .00 \\
\hline & 24 & 1.00 & .00 \\
\hline & 25 & 1.00 & .00 \\
\hline & 26 & 1.00 & .00 \\
\hline & 27 & 1.00 & .00 \\
\hline & 28 & 1.00 & .00 \\
\hline Total & $\mathbf{N}$ & 28 & 28 \\
\hline & Mean & 16.1071 & .0346 \\
\hline & Std. Deviation & 28.26507 & .06304 \\
\hline & Range & 109.00 & .24 \\
\hline & Std. Error of Skewness & .441 & .441 \\
\hline
\end{tabular}

a Limited to first 100 cases.

Descriptive

Descriptive Statistics

\begin{tabular}{|r|r|r|r|r|r|}
\hline & $\mathrm{N}$ & Minimum & Maximum & Mean & Std. Deviation \\
\hline NoOfPatients & 28 & 1.00 & 110.00 & 16.1071 & 28.26507 \\
Percentage & 28 & .00 & .24 & .0346 & .06304 \\
Valid N (listwise) & 28 & & & & \\
\hline
\end{tabular}

One-Sample Statistics

T-Test

\begin{tabular}{|r|r|r|r|r|}
\hline & & & & $\begin{array}{r}\text { Std. Error } \\
\text { Mean }\end{array}$ \\
\hline NoOfPatients & $\mathrm{N}$ & Mean & Std. Deviation & 28 \\
Percentage & 28 & $\mathbf{1 6 . 1 0 7 1}$ & $\mathbf{2 8 . 2 6 5 0 7}$ & 5.34160 \\
& .0346 & .06304 & .01191 \\
\hline
\end{tabular}

One-Sample Test

\begin{tabular}{|c|c|c|c|c|c|c|}
\hline & \multicolumn{6}{|c|}{ Test Value $=0$} \\
\hline & \multirow[b]{2}{*}{$\mathbf{t}$} & \multirow[b]{2}{*}{ df } & \multirow[b]{2}{*}{ Sig. (2-tailed) } & \multirow{2}{*}{$\begin{array}{r}\text { Mean } \\
\text { Difference }\end{array}$} & \multicolumn{2}{|c|}{$\begin{array}{r}95 \% \text { Confidence Interval of } \\
\text { the Difference }\end{array}$} \\
\hline & & & & & Lower & Upper \\
\hline NoOfPatients & 3.015 & 27 & .006 & 16.10714 & 5.1471 & 27.0672 \\
\hline Percentage & 2.908 & 27 & .007 & .03464 & .0102 & .0591 \\
\hline
\end{tabular}

Two-stage Least Squares Analysis Model Description 


\begin{tabular}{|lr|r|}
\hline & Type of Variable \\
\hline Equation 1 & Percentage & dependent \\
& NoOfPatients & predictor \& instrumental \\
\hline
\end{tabular}

Model Summary

\begin{tabular}{|c|c|c|}
\hline Equation 1 & $\begin{array}{r}\text { Multiple R } \\
\text { R Square } \\
\text { Adjusted R Square } \\
\text { Std. Error of the Estimate }\end{array}$ & $\begin{array}{r}.999 \\
.998 \\
.998 \\
.003\end{array}$ \\
\hline
\end{tabular}

ANOVA

\begin{tabular}{|rr|r|r|r|r|r|}
\hline & Sum of & & & \\
& & Squares & df & Mean Square & Fig. \\
\hline Equation 1 & Regression & .107 & 1 & .107 & 14616.936 \\
& Residual & .000 & $\mathbf{2 6}$ & .000 & .000 \\
& Total & .107 & 27 & & \\
\end{tabular}

Coefficients

\begin{tabular}{|rr|r|r|r|r|r|}
\hline & \multicolumn{2}{|c|}{ Unstandardized Coefficients } & Beta & Sig. \\
\hline & (Constant) & \multicolumn{1}{|c|}{ B } & Std. Error & & \\
\hline Equation 1 & NoOfPatients & .002 & .001 & .000 & -2.111 & 120.901 \\
& & .045 & .000 \\
\hline
\end{tabular}

Graph:(4-8)- The next chart demonstrate the ultrasound findings through 232 female patients scanned in ultrasound departments through this study in the kingdom of Saudi Arabia (Eastern area) August 2007 to May 2009.

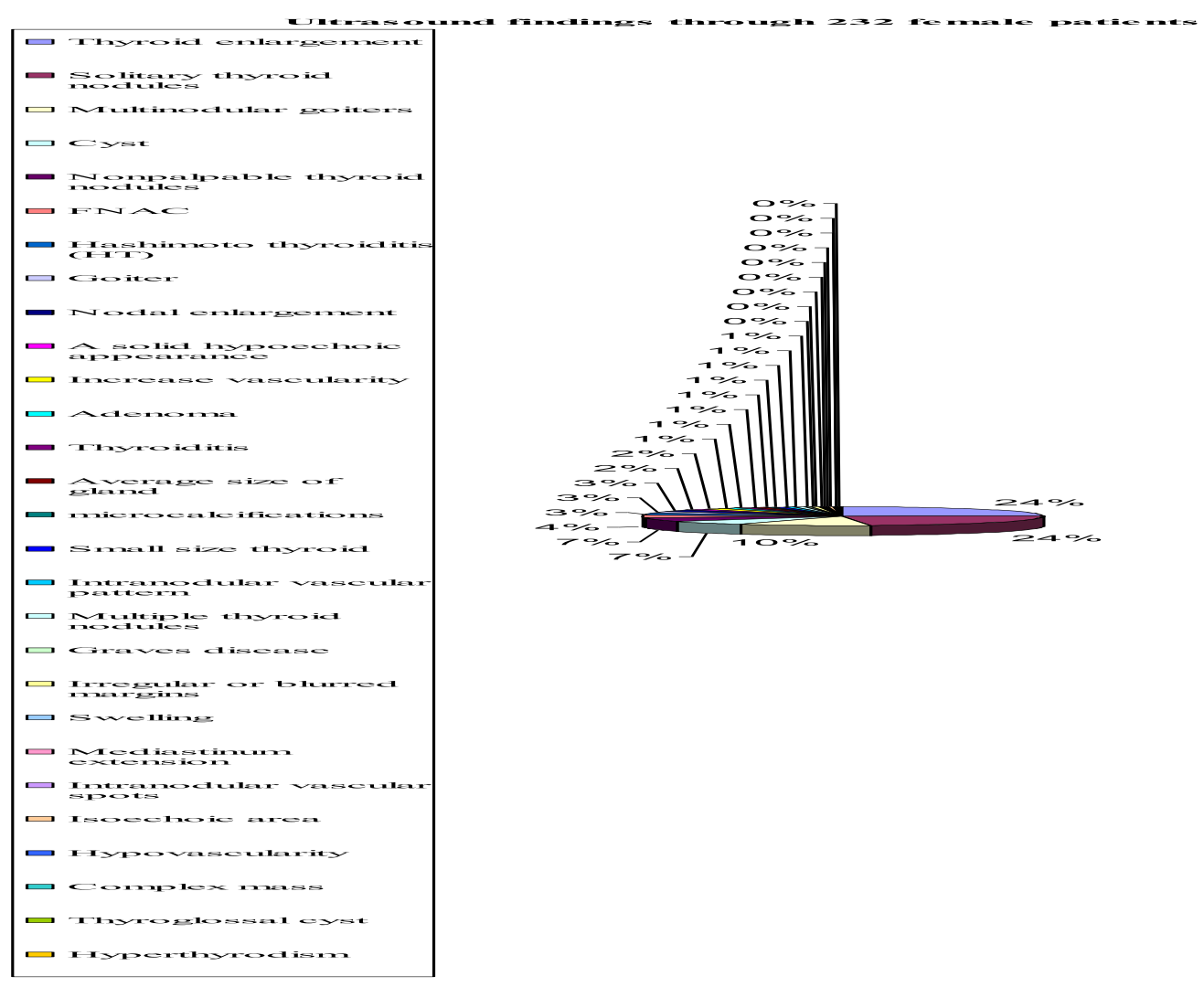


Table (4-9): Ultrasound findings through 71 male patients scanned in ultrasound departments through this study in the kingdom of Saudi Arabia (Eastern area) August 2007 to May 2009.

\begin{tabular}{|c|c|c|}
\hline Ultrasound findings & Number of patients & Percentage \\
\hline Thyroid enlargement & 37 & $27 \%$ \\
\hline Solitary thyroid nodules & 25 & $18 \%$ \\
\hline Cyst & 13 & $10 \%$ \\
\hline Nonpalpable thyroid nodules & 11 & $8 \%$ \\
\hline Goiter & 9 & $7 \%$ \\
\hline Multinodular goiters & 8 & $6 \%$ \\
\hline A solid hypoechoic appearance & 5 & $4 \%$ \\
\hline Adenoma & 5 & $4 \%$ \\
\hline Average size of gland & 4 & $3 \%$ \\
\hline FNAC & 3 & $2 \%$ \\
\hline Thyroglossal cyst & 3 & $2 \%$ \\
\hline Hashimoto thyroiditis (HT) & 3 & $2 \%$ \\
\hline Microcalcifications & 2 & $1 \%$ \\
\hline Small size thyroid & 2 & $1 \%$ \\
\hline Multiple thyroid nodules & 2 & $1 \%$ \\
\hline Intranodular vascular pattern & 1 & $1 \%$ \\
\hline Thyroiditis & 1 & $1 \%$ \\
\hline Lipoma & 1 & $1 \%$ \\
\hline Hyperplasia & 1 & $1 \%$ \\
\hline Total & 136 & $100 \%$ \\
\hline
\end{tabular}

\section{Summarize}

\section{Case Processing Summary(a)}

\begin{tabular}{|c|c|c|c|c|c|c|}
\hline & \multicolumn{2}{|r|}{ Included } & \multicolumn{2}{|r|}{ Excluded } & \multicolumn{2}{|r|}{$\begin{array}{l}\text { Cases } \\
\text { Total }\end{array}$} \\
\hline & $\mathbf{N}$ & Percent & $\mathbf{N}$ & Percent & $\mathbf{N}$ & Percent \\
\hline NoOfPatients & 20 & $100.0 \%$ & 0 & $.0 \%$ & 20 & $100.0 \%$ \\
\hline Percentage & 20 & $100.0 \%$ & $\mathbf{0}$ & $.0 \%$ & 20 & $100.0 \%$ \\
\hline
\end{tabular}

Case Summaries(a)

\begin{tabular}{|c|c|c|}
\hline & NoOfPatients & Percentage \\
\hline 1 & 37.00 & .27 \\
\hline 2 & 25.00 & .18 \\
\hline 3 & 13.00 & .13 \\
\hline 4 & 11.00 & .11 \\
\hline 5 & 9.00 & .09 \\
\hline 6 & 8.00 & .08 \\
\hline 7 & 5.00 & .05 \\
\hline 8 & 5.00 & .05 \\
\hline 9 & 4.00 & .04 \\
\hline 10 & 3.00 & .03 \\
\hline 11 & 3.00 & .03 \\
\hline 12 & 3.00 & .03 \\
\hline 13 & 2.00 & .02 \\
\hline 14 & 2.00 & .02 \\
\hline 15 & 2.00 & .02 \\
\hline 16 & 1.00 & .01 \\
\hline 17 & 1.00 & .01 \\
\hline 18 & 1.00 & .01 \\
\hline 19 & 1.00 & .01 \\
\hline 20 & 136.00 & 1.00 \\
\hline
\end{tabular}




\begin{tabular}{|r|r|r|} 
Total & 20 & 20 \\
Mean & 13.6000 & .1095 \\
Std. Deviation & 30.20526 & .22004 \\
Range & 135.00 & .99 \\
Std. Error of Skewness & .512 & .512 \\
\hline
\end{tabular}

a Limited to first 100 cases.

Descriptive Statistics

Descriptive

\begin{tabular}{|r|r|r|r|r|r|}
\hline & $\mathrm{N}$ & Minimum & Maximum & Mean & Std. Deviation \\
\hline NoOfPatients & 20 & 1.00 & 136.00 & 13.6000 & 30.20526 \\
Percentage & 20 & .01 & 1.00 & .1095 & .22004 \\
Valid N (listwise) & 20 & & & & \\
\hline
\end{tabular}

One-Sample Statistics

\begin{tabular}{|c|c|c|c|c|}
\hline & $\mathbf{N}$ & Mean & Std. Deviation & $\begin{array}{r}\text { Std. Error } \\
\text { Mean }\end{array}$ \\
\hline NoOfPatients & 20 & 13.6000 & 30.20526 & 6.75410 \\
\hline Percentage & 20 & .1095 & .22004 & .04920 \\
\hline
\end{tabular}

One-Sample Test

\begin{tabular}{|c|c|c|c|c|c|c|}
\hline & \multicolumn{6}{|c|}{ Test Value $=0$} \\
\hline & \multirow[b]{2}{*}{$\mathbf{T}$} & \multirow[b]{2}{*}{ df } & \multirow[b]{2}{*}{ Sig. (2-tailed) } & \multirow{2}{*}{$\begin{array}{r}\text { Mean } \\
\text { Difference }\end{array}$} & \multicolumn{2}{|c|}{$\begin{array}{r}\text { 95\% Confidence Interval of } \\
\text { the Difference }\end{array}$} \\
\hline & & & & & Lower & Upper \\
\hline NoOfPatients & 2.014 & 19 & .058 & 13.60000 & -.5365 & 27.7365 \\
\hline Percentage & 2.226 & 19 & .038 & .10950 & .0065 & .2125 \\
\hline
\end{tabular}

Two-stage Least Squares Analysis

Model Description

\begin{tabular}{|rr|r|}
\hline & & Type of Variable \\
\hline Equation 1 & Percentage & dependent \\
& NoOfPatients & predictor \& instrumental \\
\hline
\end{tabular}

MOD_16

\begin{tabular}{|r|r|} 
& \multicolumn{2}{|c|}{ Model Summary } \\
\hline Equation 1 & Multiple R \\
R Square & .999 \\
Adjusted R Square & .998 \\
Std. Error of the Estimate & .998 \\
& .010 \\
\hline
\end{tabular}

ANOVA

\begin{tabular}{|rr|r|r|r|r|r|}
\hline & Sum of & df & Mean Square & F & Sig. \\
\hline Equation 1 & Regression & .918 & 1 & .918 & $\mathbf{8 5 8 0 . 2 5 6}$ \\
& Residual & .002 & 18 & .000 & .000 \\
& Total & .920 & 19 & & \\
\end{tabular}

Coefficients 


\begin{tabular}{|rr|r|r|r|r|r|}
\hline & & Unstandardized Coefficients & Beta & t & Sig. \\
\hline & & B & Std. Error & & & \\
\hline Equation 1 & (Constant) & .011 & .003 & & 4.134 & .001 \\
& NoOfPatients & .007 & .000 & .999 & 92.630 & .000 \\
\hline
\end{tabular}

Graph:(4-9)- The next chart demonstrate the ultrasound finding through 71 male patients scanned in ultrasound departments through this study in the kingdom of Saudi Arabia (Eastern area) August 2007 to May 2009.
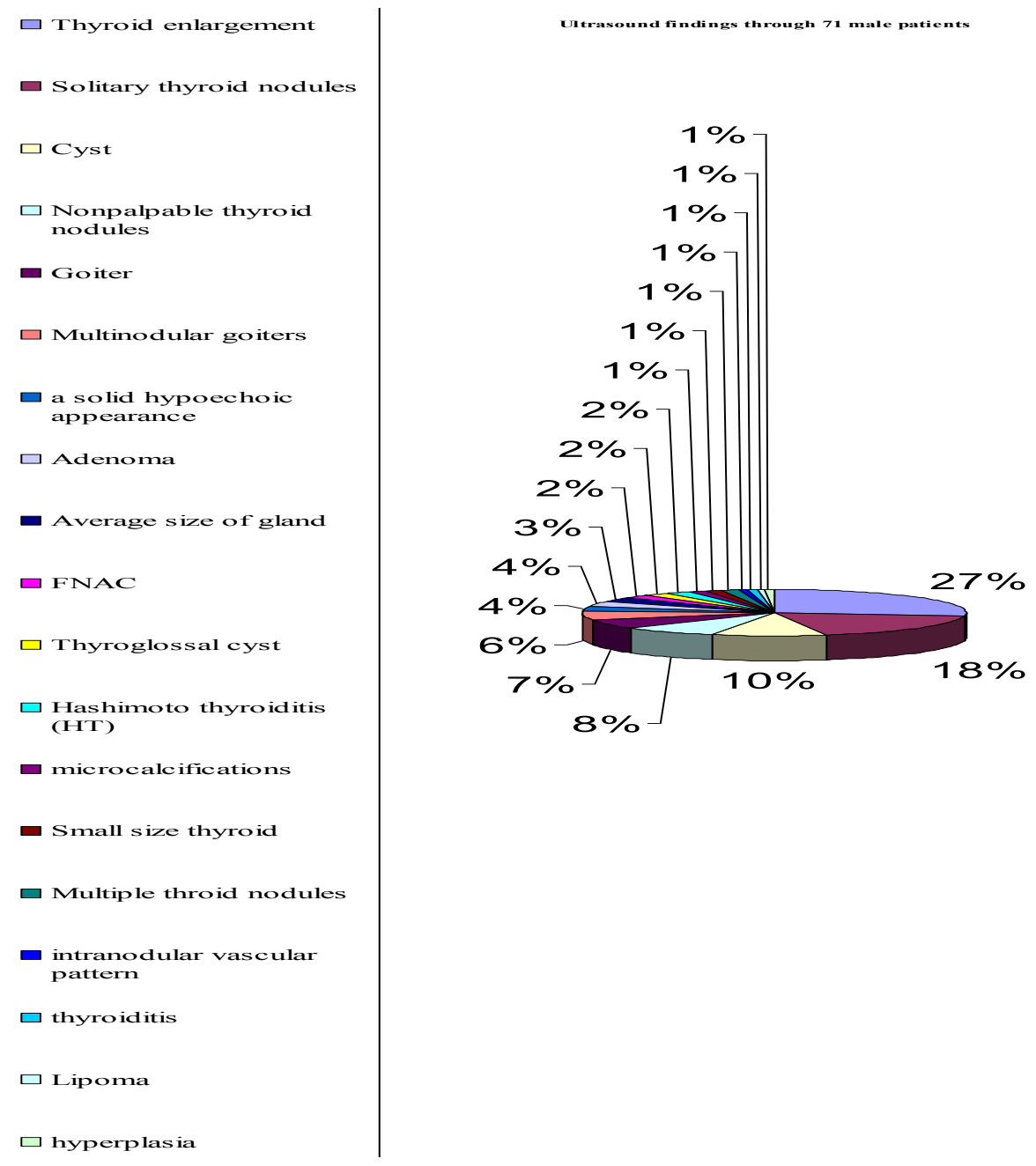

\section{Discussion}

Thyroid ultrasound is an imaging modality to evaluate the size, shape, and abnormalities of the thyroid gland.

This study which was carried out on 303 patients in which different cases of thyroid pathologies were identified sonographically, $77 \%$ were female patients and $23 \%$ were male patients (Table 4-1). So females were more affected than the males.

Previous study confirmed that, all thyroid disorders are much more common in women than in men[1]. Along this series $25 \%$ of ultrasound findings were thyroid enlargement(Figure1) which represent the most common ultrasound findings of thyroid pathologies (Table 4-5) followed by, $23 \%$ solitary thyroid nodules(Figure2), $10 \%$ multinodular goiters, $8 \%$ simple cyst(Figure3), $7 \%$ nonpalpable thyroid nodules, $4 \%$ goiter, $4 \%$ FNAC, $3 \%$ Hashimoto thyroiditis(Figure4), $2 \%$ a solid hypoechoic appearance, $2 \%$ nodal enlargement, $2 \%$ adenoma, $2 \%$ increase vascularity, $2 \%$ average size of gland, $2 \%$ thyroiditis, $1 \%$ microcalcifications, $1 \%$ small size thyroid, $1 \%$ intranodular vascular pattern, $1 \%$ multiple thyroid nodules, 1 $\%$ thyroglossal cyst and $1 \%$ Graves disease. There was no percentage presented with irregular or blured 
margins of ultrasound findings, swelling, mediastinum extension, intranodular vascular spots, isoechoic area, hypovascularity, complex mass, lipoma, hyperplasia nor hyperthyrodism. Previous study confirmed that, the majority of nonpalpable thyroid tumors can be identified by cytological evaluation of lesions presenting hypoechoic appearance in conjunction with one independent risk factor. Due to the nonnegligible prevalence of extracapsular growth and nodal metastasis, US-FNA should be performed on all 8-15 mm hypoechoic nodules with irregular margins, intranodular vascular spots or microcalcifications. Nonpalpable lesions of the thyroid without risk factors should be followed by means of clinical and US evaluation[2].

The youngest patient in this study was 55 days old boy with clinical findings of hypothyroidism, ultrasound found that nonpalpable thyroid nodule. The eldest patient was 88 years old female with thyroid enlargement. The highest incidence of thyroid pathologies between $3^{\text {rd }}$ and $5^{\text {th }}$ decades of life. While the lowest incidence in $1^{\text {st }}$ and $2^{\text {nd }}$ decades, 8 and $9^{\text {th }}$ (Table 4-2).

Previous study confirmed that, the detection of thyroid calcifications by sonography was diagnostically valuable, especially in cases involving a solitary nodule or a young person. The presence of calcifications should raise the suspicion of malignancy. The low incidence of cancer in patients with multiple noncalcified thyroid nodules suggests that a more conservative approach may be appropriate in such cases[3].

The most clinical presentation in this study (Table 4-4) associated with thyroid pathologies in this study was pain $(32 \%)$, and hyperthyroidism (19\%) they were more frequently associated with thyroid pathologies, there were 79 patients asymptomatic (Table 4-3) commonly associated with thyroid pathology.

Sonographic evaluation of thyroid pathologies revealed that thyroid enlargement and thyroid nodules were most commonly seen in thyroid pathologies and limited mass, lipoma, hyperplasia and hyperthyroidism.

According to the different signs and symptoms through 172 symptomatic female patients (Table 4-6) there were (57 of 75) were complain of pain, (36 of 45) were hyperthyroidism, (22 of 35) were neck swelling, (22 of 28) were goiter, (10 of 11) were problems with swallowing or inappropriate fixation of the neck, ( 9 of 11) were tenderness, (7 of 7) were compression of the respiratory tract, $(8$ of 10) were hypothyroidism, (6 of 9) were thyroid nodule, (2 of 2) were vocal fold paralysis, (2 of 2) were thyrotoxic, (1 of 1$)$ was enlarge thyroid, ( 1 of 1$)$ was sweating, ( 1 of 1$)$ was nervous and anxiety, ( 1 of 1$)$ was fullness of neck, ( 1 of 1$)$ was thyroiditis, ( 1 of 1$)$ was adenomyopathy elevated T3, T4 and TSH, (1 of 1) was Graves disease . Previous study confirmed that, Ultra- sound can obviate the need for scintigraphy in more than half (54\%) of patients with possible congenital hypothyroidism. Ultrasound had a potential to predict prognosis of patients with possible congenital hypothyroidism[4].

Usually the nodule are solitary but may be multiple. As was seen in this study, (23\%) cases presented solitary thyroid nodules, (1\%) cases as multiple thyroid nodules (Table 4-5).

Thyroid enlargement and solitary thyroid nodules were the most common concomitant with thyroid pathology during this study (147 and 131patients) (Table 4-5).23 cases were aspirated under ultrasound guidance without any mentioned of complications or side effect, (15 of 23) were sent to the laboratory for cystological examination, (1 of 23) was chronic lymphocytic thyroiditis (Hashimoto thyroiditis (HT)), (1of 23) was thyroid nodule (Benign lesion), (1 of 23) was nodular goiter observed in autoimmune thyroid disease (AITD), (4 of 23) were thyroid cyst. Previous study confirmed that, two drawbacks were noted when conventional FNAB was used: (1) cancer lesions difficult to palpate (e.g., small cancers with or without benign lesions or cancers associated with Hashimoto's thyroiditis or Graves' disease); and (2) palpable cancers with insufficient cell material for analysis (e.g., cystic carcinoma and cancers with calcified lesions)[5]. UG-FNAB is a powerful technique for detecting microcancers, cystic carcinomas, cancers associated with benign nodules, Hashimoto's thyroiditis, or coarse calcifications[5]. High-resolution ultrasonography is sensitive and capable of detecting many small, nonpalpable thyroid nodules. Most of these lesions are benign. For most patients with nonpalpable nodules that are incidentally detected by thyroid imaging, simple follow-up neck palpation is sufficient[6]. Abnormal imaging studies suggesting thyroid pathology are almost invariably followed by ultrasonography of the thyroid. Endocrinologists themselves are using ultrasonography more frequently. A recent study suggested that ultrasonography would alter the clinical management of nodular thyroid disease in $63 \%$ of patients [7]. 


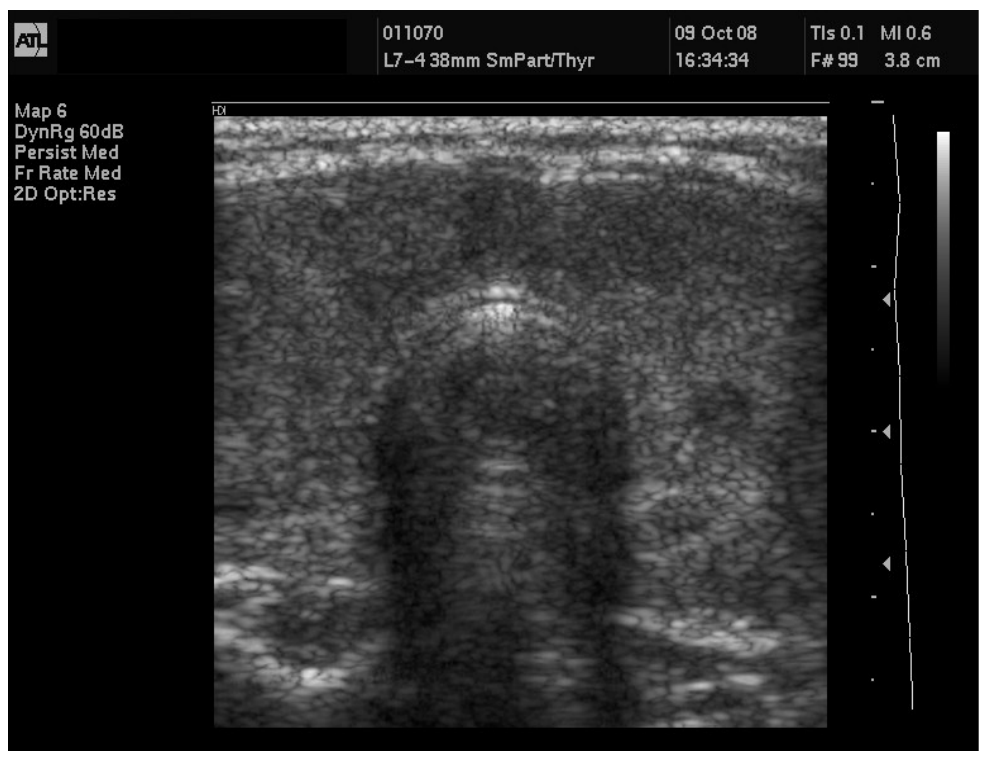

Figure 1: Female 31 years old; thyroid enlargement and multinodular goiters.

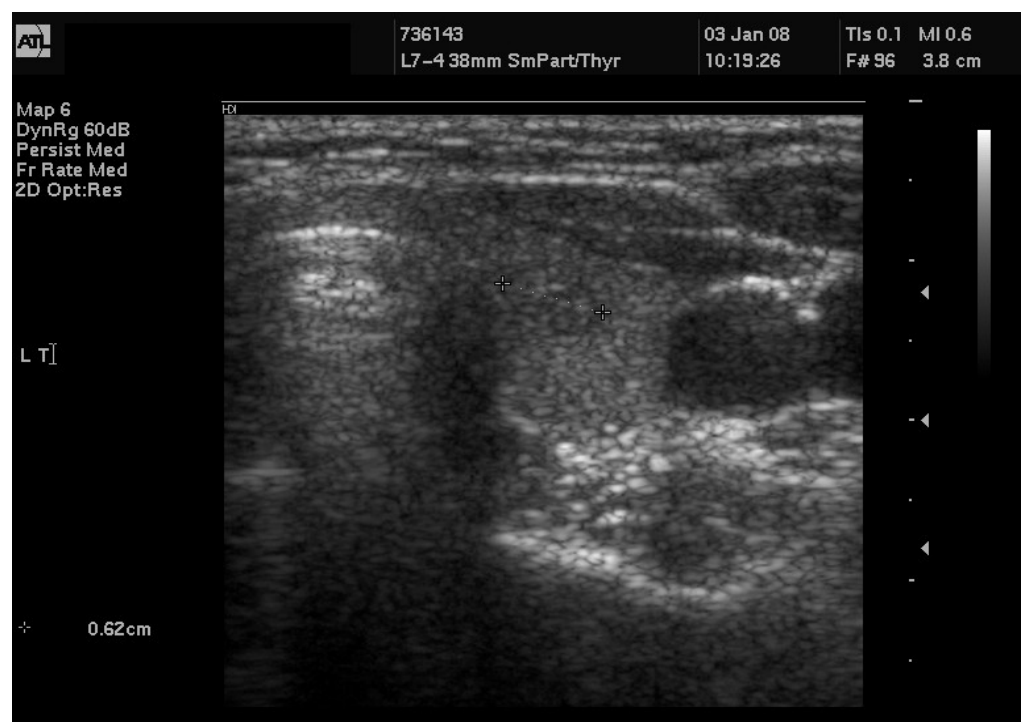

Figure 2: Female 55 years old; left solitary thyroid nodule $(0.6 \mathrm{x} 0.6) \mathrm{cm}$

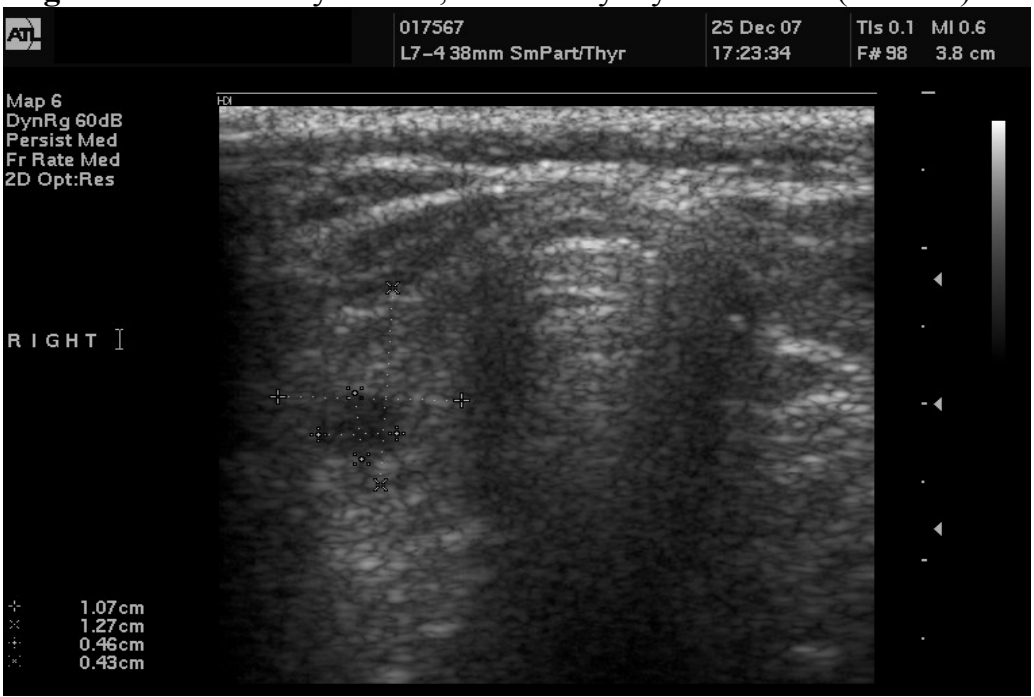

Figure3:Female 77 years old; right thyroid lobe small cyst. 


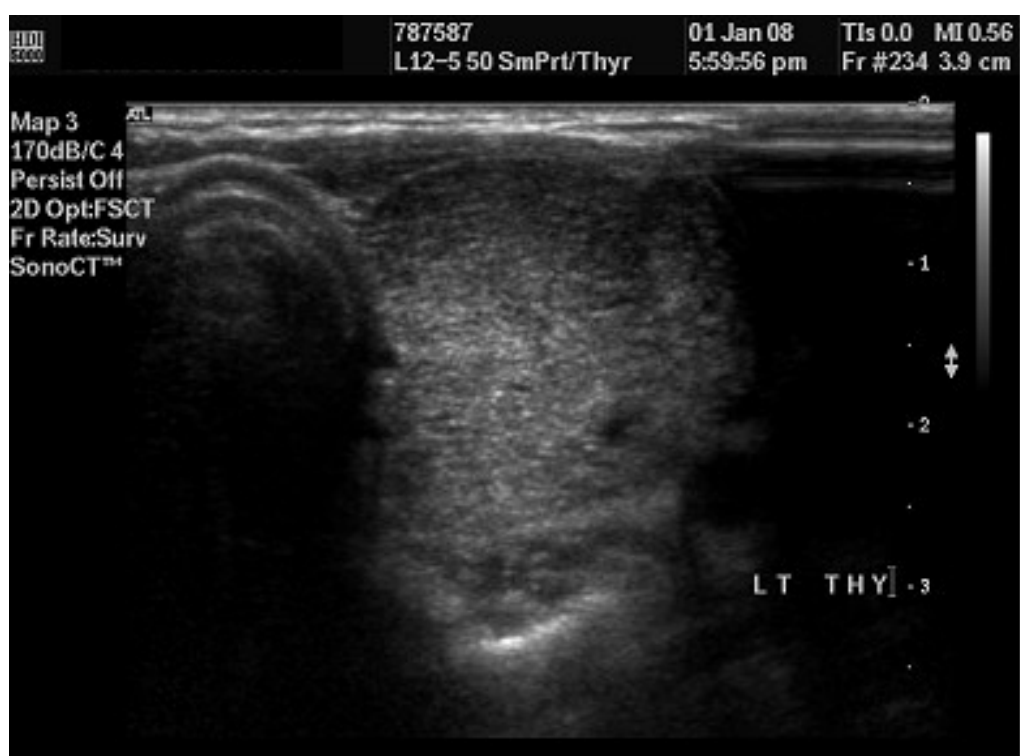

Figure4: Female 25 years old; bilateral diffuse thyroiditis (Hashimoto's disease).

\section{VI. conclusion}

Sonography is the single cost effective mean in detection of thyroid pathologies.

Ultrasound -guided aspiration is an expeditious mean to helps to identify a bacterial cause of the nodule.

This study has shown one case of complex mass, lipoma, hyperplasia and hyperthyroidism.

The highest incidence of thyroid diseases in between $3^{\text {rd }}$ and $5^{\text {th }}$ decades of life. While the lowest incidence in $1^{\text {st }}$ and $2^{\text {nd }}$ decades $/ 8$ and $9^{\text {th }}$ decades of life. All thyroid disorders are much more common in women than in men. In conclusion, the thyroid gland pathologies obtained in this study was agreement with a previous study.

\section{Recommendations}

Believe more that diagnostic Ultra sonography should be the first-line test owing to its safety and availability. The recommendations in this consensus statement, which are based on analysis of the current literature and common practice strategies, are thought to represent a reasonable approach to thyroid pathologies. Thyroid US is the imaging method of choice for the evaluation of thyroid gland structure, and FNAC, as the most accurate test for nodule diagnosis, has reduced the need for scanning and for thyroidectomy, thereby reducing the healthcare costs significantly.

The clinical management of thyroid diseases and to propose a work-up which is very likely to diagnose benign or malignant thyroid neoplasia, preoperatively. The standard diagnostic protocol of thyroid diseases consists of: (i) patient's history including the prior existence and treatment of a benign thyroid disease, (ii) clinical examination, (iii) laboratory tests, (iv) thyroid ultrasound (US), (v) scintigraphy (SC), (vi) fine-needle aspiration biopsy (FNAB) and (vii) molecular studies employed for the detection of malignancy as a part of clinical research.

[1] http://www.thyroid.ca/Guides/HG01.html.

\section{References}

[2] http://www.springerlink.com/content/3732n0ewhqb6amd7/

[3] http://jcem.endojournals.org/cgi/content/full//87/5/1938

[4] http://www3.interscience.wiley.com/cgi-bin/abstract/72514684/ABSTRACT

[5] http://www.ajnr.org/cgi/content/abstract/16/5/1117

American Journal of Neuroradiology, Vol 16, Issue 5 1117-1123, Copyright (C) 1995 by American Society of Neuroradiology.

[6] http://www.springerlink.com/content/4h43yxr7ay7xwna8/

[7] Jarlov AE, Hegedus L, Gjorup T, Hansen JEM. Accuracy of the clinical assessment of the thyroid size. Dan Med Bull 1991; 38:87-89.

\section{DATA COLLECTION SHEET (QUESTIONNAIRE)}

\begin{tabular}{|c|c|c|c|c|c|}
\hline \multicolumn{4}{|c|}{ Sex......Age.......... Hospital............. I.D...... Refer Dr/Dept... } & r/Dept... & \\
\hline Clinical Findings & $\begin{array}{l}\sqrt{ } / \\
\mathbf{x}\end{array}$ & Ultrasound Findings & $\begin{array}{l}\sqrt{ } \\
\text { / } \\
\mathbf{x}\end{array}$ & $\begin{array}{l}\text { Aspirate } \\
\text { (FNAC) }\end{array}$ & $\begin{array}{l}\sqrt{ } \\
\text { l } \\
\mathbf{X}\end{array}$ \\
\hline Asymptomatic & & Thyroid enlargement & & $\begin{array}{l}\text { 1-Nodular goiter observed in autoimmune thyroid } \\
\text { disease (AITD), i.e. }\end{array}$ & \\
\hline
\end{tabular}


Role Of Ultrasound In Thyroid Pathologies

\begin{tabular}{|c|c|c|}
\hline Pain & Nonpalpable thyroid nodules & $\begin{array}{l}\text {-chronic lymphocytic thyroiditis (Hashimoto thyroiditis } \\
\text { (HT)) }\end{array}$ \\
\hline Tenderness & Solitary thyroid nodules & -Graves' disease (GD) \\
\hline $\begin{array}{l}\text { Compression of the } \\
\text { respiratory tract }\end{array}$ & Multinodular goiters & -nonpalpable thyroid nodules \\
\hline $\begin{array}{l}\text { Problems with } \\
\text { swallowing or } \\
\text { inappropriate fixation } \\
\text { of the neck }\end{array}$ & Mediastinum extension & 2-Folicular adenoma \\
\hline Vocal fold paralysis & Nodal enlargement & 3-Cyst \\
\hline $\begin{array}{l}\text { Heat and redness of } \\
\text { the skin over the } \\
\text { nodule }\end{array}$ & Neoplastic lesions & 4-Papillary carcinoma \\
\hline \multirow[t]{6}{*}{ Others.............. } & a solid hypoechoic appearance & \multirow{6}{*}{ Others : } \\
\hline & irregular or blurred margins & \\
\hline & intranodular vascular pattern & \\
\hline & Microcalcifications & \\
\hline & intranodular vascular spots & \\
\hline & Others............ & \\
\hline
\end{tabular}

